\title{
Inversiones térmicas con advección cálida inferior en la Vega Media del Segura (Región de Murcia)
}

\section{Temperature Inversions due to warm air advections at low levels in the Vega Media del Segura river (Region of Murcia)}

\author{
Carmelo Conesa García*, David Espín Sánchez, Ramón García Marín, \\ Gregorio Castejón Porcel y Daniel Moreno Muñoz
}

\section{INTRODUCCIÓN}

El origen y desarrollo del embolsamiento de aire frío en niveles bajos de la atmósfera, a menudo próximos a la superficie, se hallan generalmente asociados a periodos de baja radiación solar, alta presión atmosférica y vientos suaves (Barr and Orgill, 1989; Beniston, 2006; Lundquist and Cayan, 2007). Bajo tales condiciones sinópticas un balance de radiación negativo implica un enfriamiento rápido de la superficie y la formación de una capa de aire baja estable (Whiteman, 2000). Las laderas de un valle o de una depresión interior refuerzan el proceso cuando actúan flujos catabáticos, siendo tanto más acusado cuanto mayor es el confinamiento de las tierras bajas (Gustavsson et al., 1998; Lindkvist et al., 2000; Lundquist et al., 2008). Como resultado se producen fenómenos de inversión térmica (IT) cuya intensidad y espesor varían de acuerdo con la situación sinóptica, la persistencia del balance energético negativo y la configuración topográfica del valle (McKee and O'Neal, 1989; Whiteman, 2000; Clements et al., 2003; Daly et al., 2009). La falta de mezcla vertical dentro de estas inversiones térmicas de valle aísla una porción de aire

\footnotetext{
*Departamento de Geografía, Facultad de Letras, Universidad de Murcia (cconesa@um.es).
} 
inferior respecto de la atmósfera libre superior (Whiteman, 2000). En tales casos, las inversiones por advección o invasión horizontal, por debajo de aire frío, o por encima del cálido, son más frecuentes, extensas e importantes que las de irradiación (Trewartha, 1943).

La mayor parte de la producción científica alude a situaciones de inversión térmica en zonas de valle (ITV); en cambio es más escasa la referente a estancamientos de aire frío en cuencas fluviales (ITC). Determinados sectores, dentro de éstas, pueden aparecer cubiertos en invierno por nubes bajas, que disminuyen la insolación y favorecen la formación de bolsas de aire frío más o menos persistentes en los niveles inferiores (Petkovsek, 1974; Ishikawa 1977; Kondo y Okusa 1990; Kuwagata y Sumioka 1991). El problema se agrava cuando las inversiones térmicas vienen impuestas o condicionadas por la ocurrencia de episodios de polución (Incecik 1996; Kukkonen et al. 2005; Malek et al. 2006). En muchas ciudades europeas, tanto las inversiones en altura como las de niveles inferiores, próximas a la superficie, coinciden con periodos de alta concentración de aerosoles (Kukkonen et al., 2005; Bailey et al., 2011). En España, el estudio de las inversiones térmicas empieza a tener cierto desarrollo a partir de mediados del siglo xx. En las décadas de los 60 y 70 se aborda el estudio de masas de aires sometidas a flujos térmicos de irradiación o advectivos, que alteran la estructura vertical de los niveles bajos de la atmósfera. En unos casos, se analizan heladas con advección fría y régimen anticiclónico caracterizadas por una fuerte inversión térmica en las capas inmediatas al suelo (Albero y Catalá, 1965; Puigdefábregas, 1970). En otros, como el investigado por López Gómez (1975) entre Madrid y la Sierra de Guadarrama, se describen inversiones de temperatura con advección cálida en niveles atmosféricos más elevados.

Desde los años ochenta hasta la actualidad abundan los estudios de ámbito local, algunos de gran repercusión y referencia obligada: e.g. Gesteiro Araujo y Galán Gallego (1983) sobre inversiones térmicas invernales en el sector central de la Serranía de Cuenca; Xercavins (1989) sobre IT en la subcomarca de Les Guilleries (Cataluña); Martí Ezpeleta (1990) en la Depresión de Sariñena; Martín Vide y Fructuoso Aranda (1993) en el Vallès Oriental; Dorta Antequera (1994) en Canarias, Domínguez Bascón (1995) en el valle del Guadalquivir, Bello (2008) en el Valle Bajo del río Henares, y Batlló (2009) en la Plana de Vic.

En esta línea se enmarca el presente artículo, que tiene por objeto principal el estudio de inversiones térmicas con advección cálida inferior en la Vega Media del Segura. Por ser éste un espacio intensamente ocupado y de gran tradición hortofrutícola, el análisis de condiciones térmicas extremas adquiere aquí especial relevancia. La vulnerabilidad de este tipo de cultivos (e.g. cítri- 
cos, hortalizas, etc.) ante el rigor de temperaturas excesivamente bajas en ciertos momentos del ciclo vegetativo hace necesario conocer la magnitud y frecuencia de las heladas de inversión térmica en el área. El fuerte descenso de las temperaturas nocturnas durante el periodo invernal, especialmente en situaciones de total estabilidad, es un hecho relativamente frecuente en dicha Vega. Dentro de este contexto resultan claves ciertas configuraciones sinópticas que favorecen procesos de acusada inversión térmica cuando por lo general las temperaturas medias mensuales son suaves durante casi todo el año, incluso en época invernal. La aparición de la dorsal subtropical marítima en invierno, con predominio de tiempo estable en el Sureste español, determina altas temperaturas diurnas, pero también fuertes descensos térmicos por la noche que originan importantes heladas (Olcina Cantos y Rico Amorós, 1995). Tales situaciones serán analizadas, junto con diversos factores locales, para completar la información existente y mejorar el conocimiento sobre el desarrollo de inversiones térmicas en la Vega Media del Segura, y, con ello, en los valles prelitorales del Sistema Bético.

\section{ÁREA DE ESTUDiO}

Como área de estudio se ha elegido la Vega Media del Segura y sus estribaciones, situada en el sector oriental de la Región de Murcia, y en gran parte dentro de un largo valle-corredor conocido como la Depresión Prelitoral Guadalentín - Bajo Segura (Sureste peninsular). Ésta es una depresión post-orogénica, rellena de materiales neógenos y cuaternarios (López Bermúdez et al., 1986), con altitudes que varían entre los 30 y $100 \mathrm{~m}$ (figura 1). Al norte limita con una serie de elevaciones modestas, de cotas inferiores a $200 \mathrm{~m}$ (cerros de Espinardo, Cabezo de Torres y Esparragal), y al sur con la parte oriental de la Sierra de Carrascoy, más compacta y escarpada (Cabezo del Puerto, 528 m; Relojero, $600 \mathrm{~m}$ ). El fondo del valle es recorrido por el Bajo Segura, cuyos continuos aportes sedimentarios en épocas de crecidas ha originado una extensa llanura aluvial (Lillo Carpio, 2000).

La Vega Media del Segura cuenta con un gran número de días despejados y de horas de sol al año, lo que unido al predominio de situaciones anticiclónicas, particularmente en periodo invernal, genera importantes y frecuentes inversiones térmicas por irradiación nocturna (Calvo García-Tornel, 1982; Conesa García y Alonso Sarría, 2006). La temperatura media anual se sitúa en torno a $18{ }^{\circ} \mathrm{C}$, las medias de máximas en los meses de verano superan los $32{ }^{\circ} \mathrm{C}$, y las medias de mínimas en los meses de invierno apenas alcanzan 
FIGURA 1

\section{LOCALIZACIÓN DEL ÁREA DE ESTUDIO Y DE LAS ESTACIONES METEOROLÓGICAS UTILIZADAS, CON INDICACIÓN \\ DE PERFILES TOPOGRÁFICOS}

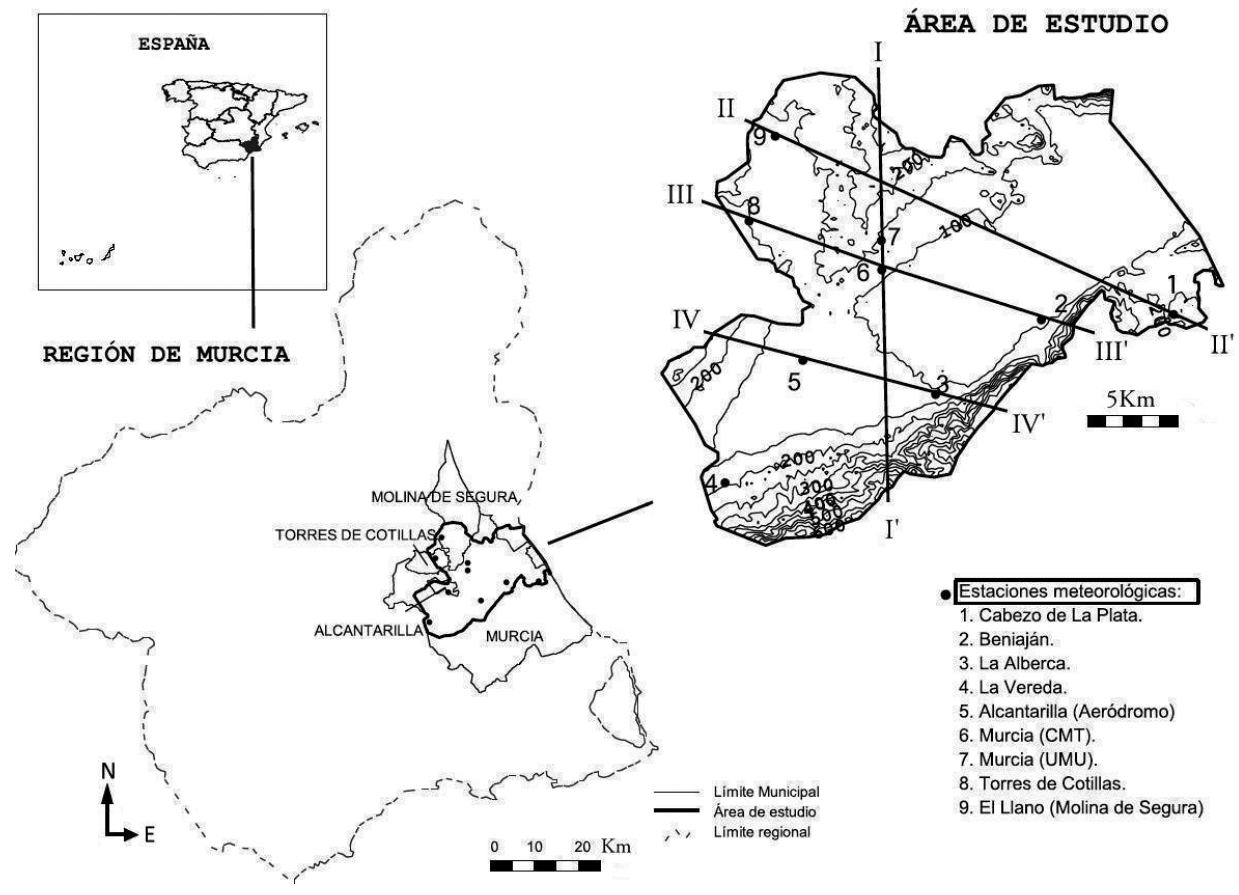

Fuente: elaboración propia.

los $5^{\circ} \mathrm{C}$. La precipitación anual es escasa (300 a $350 \mathrm{~mm}$ ) y de extrema irregularidad (Geiger, 1973). Con 442.403 habitantes (INE, 2011), la mayoría residentes en la ciudad de Murcia, esta área es la más poblada de la Región. Las excelentes condiciones agronómicas de sus suelos y la bonanza climática durante gran parte del año han llevado a la población de la Vega a basar su economía tradicional en la actividad agrícola. Las cosechas de plantaciones hortícolas y frutales cítricos han sido exportadas a numerosos países de toda Europa, generando un sector económico de gran importancia. En los últimos años, dicha actividad agrícola he decrecido de forma significativa (García Martín, 2012), aunque todavía existen muchas pequeñas explotaciones de regadío familiares, que pueden verse afectadas por heladas ocasionales. 
FIGURA 2

REPRESENTACIÓN DE LOS PERFILES TOPOGRÁFICOS TRAZADOS EN LA FIG. 1
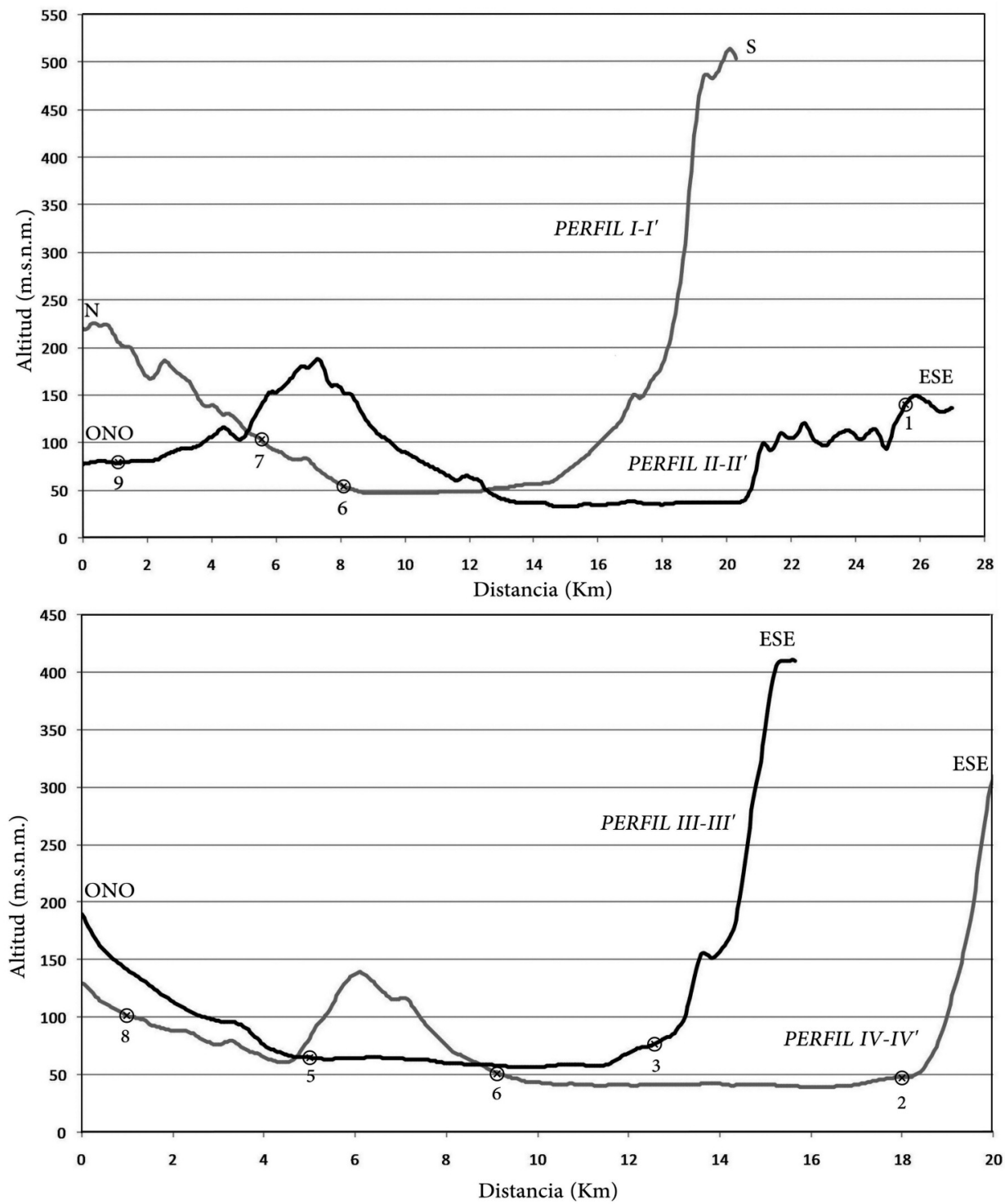

Fuente: elaboración propias. 
Los perfiles topográficos son una clara muestra gráfica de la disposición y forma del valle del Segura en su Vega Media (figura 2). Representan una depresión de fondo plano, producto del desarrollo de una extensa llanura aluvial, con orientación inicial N-S, que a pocos $\mathrm{km}$ cambia a OSO-ESE, siguiendo la dirección principal de la Depresión Prelitoral. Al S y ESE se alza la Sierra de Carrascoy, que sirve de canalizadora de los vientos del OSO, al tiempo que retiene los flujos fríos del N. Las estaciones situadas a cotas más bajas son Alcantarilla, Murcia CMT, La Alberca y Beniaján, mientras que las más expuestas a los vientos del $\mathrm{N}$ se hallan en el Llano de Molina y Torres de Cotillas.

\section{Metodología}

El presente estudio se inicia con un análisis en superficie de las variables climáticas normalmente involucradas en el fenómeno de inversión térmica (e.g. temperaturas mínimas medias y mínimas absolutas diarias, amplitudes térmicas diarias, dirección y velocidad del viento). Para dicho análisis se han utilizado las series de datos meteorológicos diarios, correspondientes al período 2000-2011, de un total de 9 observatorios ubicados en el área de estudio (tabla 1). Tales estaciones pertenecen a las redes del SIAM-IMIDA (Sistema de Información Agro-meteorológica del Instituto Murciano de Investigación y Desarrollo Alimentario) y a la Agencia Estatal de Meteorología (AEMET). De las series de datos diarios empleadas (datos referentes a 4020 días), se han extraído las 20 situaciones de inversión térmica más potentes. En su estudio se han empleado también los datos de los Sondeos Atmosféricos realizados por el Centro Meteorológico Territorial (CMT) de Murcia en hora nocturna, concretamente a las 00 horas (00Z). Con esta información se ha llevado a cabo un análisis de temperatura en superficie, en relación con la temperatura del estrato cálido y los valores térmicos correspondientes a la topografía de $850 \mathrm{hPa}$.

Para delimitar los episodios de inversión térmica en las estaciones del área se han analizado los datos de los sondeos atmosféricos del observatorio de Guadalupe (CMT de Murcia). Los resultados arrojan balances térmicos positivos entre la superficie y el estrato cálido en episodios de duración siempre inferior a 12 días. Lo más frecuente es que la situación de inversión térmica (SIT) dure entre 5 y 7 días, por lo que la dispersión que pudieran enmascarar los valores medios calculados para cada episodio no es muy significativa. El día de máxima inversión térmica (MIT) no ocupa necesariamente el centro del episodio, más bien se sitúa hacia la fase final del mismo. 
TABLA 1

ESTACIONES METEOROLÓGICAS UTILIZADAS EN EL ESTUDIO

\begin{tabular}{lccccc}
\hline \multicolumn{1}{c}{$\begin{array}{c}\text { Estación } \\
\text { Meteorológica }\end{array}$} & Municipio & $\begin{array}{c}\text { Altitud } \\
(\mathrm{m})\end{array}$ & UTM X & UTM Y & Red \\
\hline Cabezo de la Plata & Murcia & 128 & 677062 & 4205239 & SIAM IMIDA \\
Beniaján & Murcia & 50 & 669751 & 4204934 & AEMET \\
La Alberca & Murcia & 54 & 663907 & 4200807 & SIAM IMIDA \\
La Vereda & Murcia & 142 & 652257 & 4195930 & SIAM IMIDA \\
$\begin{array}{l}\text { Alcantarilla } \\
\text { (Aeródromo) }\end{array}$ & Alcantarilla & 75 & 656559 & 4202697 & AEMET \\
$\begin{array}{l}\text { Murcia CMT } \\
\text { Murcia U.M.U }\end{array}$ & Murcia & 61 & 660934 & 4207685 & AEMET \\
Torres de Cotillas & Las Torres de Cotillas & 110 & 653585 & 4210381 & AEMET \\
El Llano & Molina de Segura & 80 & 655031 & 4215095 & SIAM IMIDA \\
\hline
\end{tabular}

Fuente: Datos de las redes del SIAM-IMIDA y de la AEMET.

De hecho, la curva de intensidad de dichas SIT es generalmente disimétrica, con un limbo ascendente de pendiente moderada o suave y una rama descendente más brusca.

La caracterización de este fenómeno ha sido completada mediante la relación de variables relativas a la variación térmica espacial (superficial y vertical) dentro del área de estudio (e.g. diferencias máximas entre fondo de valle y zona externa a la Vega de temperaturas mínimas en superficie versus las de amplitud térmica diaria constatadas los días de MIT; incremento de temperatura entre superficie y estrato cálido versus temperatura a $850 \mathrm{hPa}$ durante los días de MIT). Estadísticamente se comprueba qué tipo de ajuste teórico por regresión presenta más fiabilidad (i.e. se establecen las funciones que explican dichas relaciones), y se muestran las ecuaciones de tendencia para cada caso.

Por último, se ha realizado un detallado análisis sinóptico de las 3 situaciones de inversión térmica más destacadas y de su evolución atmosférica. Dicho análisis se basa en la interpretación de mapas sinópticos del Boletín Meteorológico, elaborados por el antiguo INM (Instituto Nacional de Meteorología), actual AEMET, y de mapas proporcionados por el NCEP (NOAA) (National 
Centre Environmental Prediction-National Oceanic and Atmospheric Administration), disponibles en el archivo de la web Wetterzentrale, con análisis de isobaras en superficie y topografía térmica a $850 \mathrm{hPa}$.

\section{RESULTADOS ANALÍTICOS}

En noches claras y diáfanas, con total estabilidad, tras la puesta de sol, se produce el enfriamiento de la superficie terrestre debido a la pérdida de calor por radiación neta. Su temperatura cae por debajo de la que ofrece la masa de aire en contacto con ésta y enfría las capas de aire contiguas superiores por conducción. Éstas registran una densidad cada vez mayor, en oposición al estrato que queda por encima, cuyos valores térmicos son superiores a los de superficie. Además, al existir un área montañosa receptora de aire frío, como la Sierra de Carrascoy, se produce el descenso catabático de dicho aire, más denso, a través de su ladera. Esta nueva masa, a pesar de calentarse ligeramente, cede energía calorífica a la vertiente enfriada, acumulándose en el fondo de valle. Por lo general, y éste es el caso, las pérdidas de calor por radiación nocturna se sitúan en torno a $0,1 \mathrm{cal} / \mathrm{cm}^{2}$ para suelos desnudos, mientras que el contenido de humedad atmosférica relativa no rebasa el 50-60\% (Hernández Navarro, 1994).

A continuación se describe el comportamiento de las variables meteorológicas más influyentes en los episodios de inversión térmica analizados (temperaturas mínimas absolutas, amplitud térmica diaria, vientos de superficie y variables obtenidas mediante sondeos aerológicos -estratificación térmica e indicadores de estabilidad atmosférica-).

\section{Temperaturas mínimas absolutas}

Las temperaturas mínimas absolutas diarias registradas en el área durante las SIT varían notablemente en muy pocos kilómetros de distancia, a pesar de no existir gran irregularidad topográfica ni marcadas diferencias altitudinales (apenas 120 m), salvo la impuesta por la citada Sierra de Carrascoy. Según Martí Ezpeleta (1990), la mayor frecuencia de inversiones térmicas nocturnas se produce en periodo invernal. En el caso de la Vega Media del Segura, de las 20 SIT más intensas en el periodo 2000-2011, quince (75\%) tienen lugar entre los meses de enero (40\%) y febrero (35\%). El resto se reparten entre diciembre (15\%) y noviembre (10\%).

De los datos analizados se infiere que las temperaturas mínimas medias más bajas se registran en las zonas más bajas, como Alcantarilla $\left(4,5^{\circ} \mathrm{C}\right)$, La 
Alberca $\left(5,0^{\circ} \mathrm{C}\right)$ y Murcia $\mathrm{CMT}\left(5,2^{\circ} \mathrm{C}\right)$, o en las más expuestas a los vientos dominantes del $\mathrm{N}$ (Llano de Molina, $1,9^{\circ} \mathrm{C}$; Las Torres de Cotillas, $3,8^{\circ} \mathrm{C}$ ). Estas temperaturas suelen ser inferiores a lo habitual (temperatura mínima media invernal de los últimos 30 años). Por el contrario, las temperaturas mínimas medias diarias más elevadas corresponden a zonas periféricas y resaltes topográficos, como es el caso de Cabezo de la Plata $\left(8,1^{\circ} \mathrm{C}\right)$, Murcia UMU $\left(7^{\circ} \mathrm{C}\right)$ y La Vereda $\left(7^{\circ} \mathrm{C}\right)$, todas ellas con valores por encima del promedio invernal (tabla 2).

Los valores absolutos de temperatura mínima diaria más bajos de toda la Vega se dan también en el Llano de Molina (El Llano), sector topográficamente deprimido donde confluyen los ríos Mula y Segura. Esta zona de valle, rodeada por modestos rebordes montañosos, constituye una pequeña cubeta comunicada con la Huerta de Murcia a través del río Segura. Las temperaturas mínimas diarias registradas aquí son manifiestamente inferiores a las observadas en las demás estaciones meteorológicas, llegando a estar por debajo de los $0{ }^{\circ} \mathrm{C}$ en 5 SIT completas y en el $50 \%$ de los días con inversión térmica más intensa (tablas 2 y 3 ). En el 95\% de las SIT Llano de Molina ostenta el mínimo absoluto diario del área, mientras que el observatorio Torres de Cotillas lo hace sólo en el 5\% de situaciones restantes. En contraste, las temperaturas mínimas absolutas más elevadas, al igual que las medias, se registran en sectores marginales, fuera del valle y del área de dominio de las SIT. Este es el caso de las estaciones de La Vereda y Cabezo de la Plata, ambas situadas en el piedemonte noroccidental de la Sierra de Carrascoy.

Existe pues una clara diferencia entre las temperaturas mínimas diarias de las estaciones del fondo del valle, afectado de lleno por las inversiones térmicas, y las registradas en sectores marginales o de mayor altitud, donde las SIT apenas dejan notar su influencia. La media de mínimas diarias estimada para el total de episodios IT varía entre $1,9^{\circ} \mathrm{C}$ en El Llano y $8,1^{\circ} \mathrm{C}$ en el Cabezo de la Plata (diferencia de $6,5^{\circ} \mathrm{C}$ ). El contraste de los valores de temperatura en superficie entre ambas zonas, cuando ocurre este tipo de fenómeno, aumenta conforme resulta más intensa y duradera la inversión térmica. De hecho, durante la SIT más potente del período (14-24 de noviembre de 2004) se alcanzó la máxima diferencia de temperatura media de mínimas, a niveles bajos, entre las citadas estaciones: $-1,2^{\circ} \mathrm{C}$ en el Llano de Molina, frente a los $8{ }^{\circ} \mathrm{C}$ del Cabezo de la Plata (tabla 2). Considerando los días álgidos de cada episodio de inversión térmica (tabla 3) se observan valores de temperatura mínima muy diferentes entre el fondo de valle y los sectores de piedemonte, con variaciones superiores a $10^{\circ} \mathrm{C}$ en siete de las situaciones analizadas y a $13,5^{\circ} \mathrm{C}$ en dos de ellas $\left(13,6^{\circ} \mathrm{C}\right.$ el 31 de diciembre de 2001 y $14,1^{\circ} \mathrm{C}$ el 27 de febrero de 2002). 


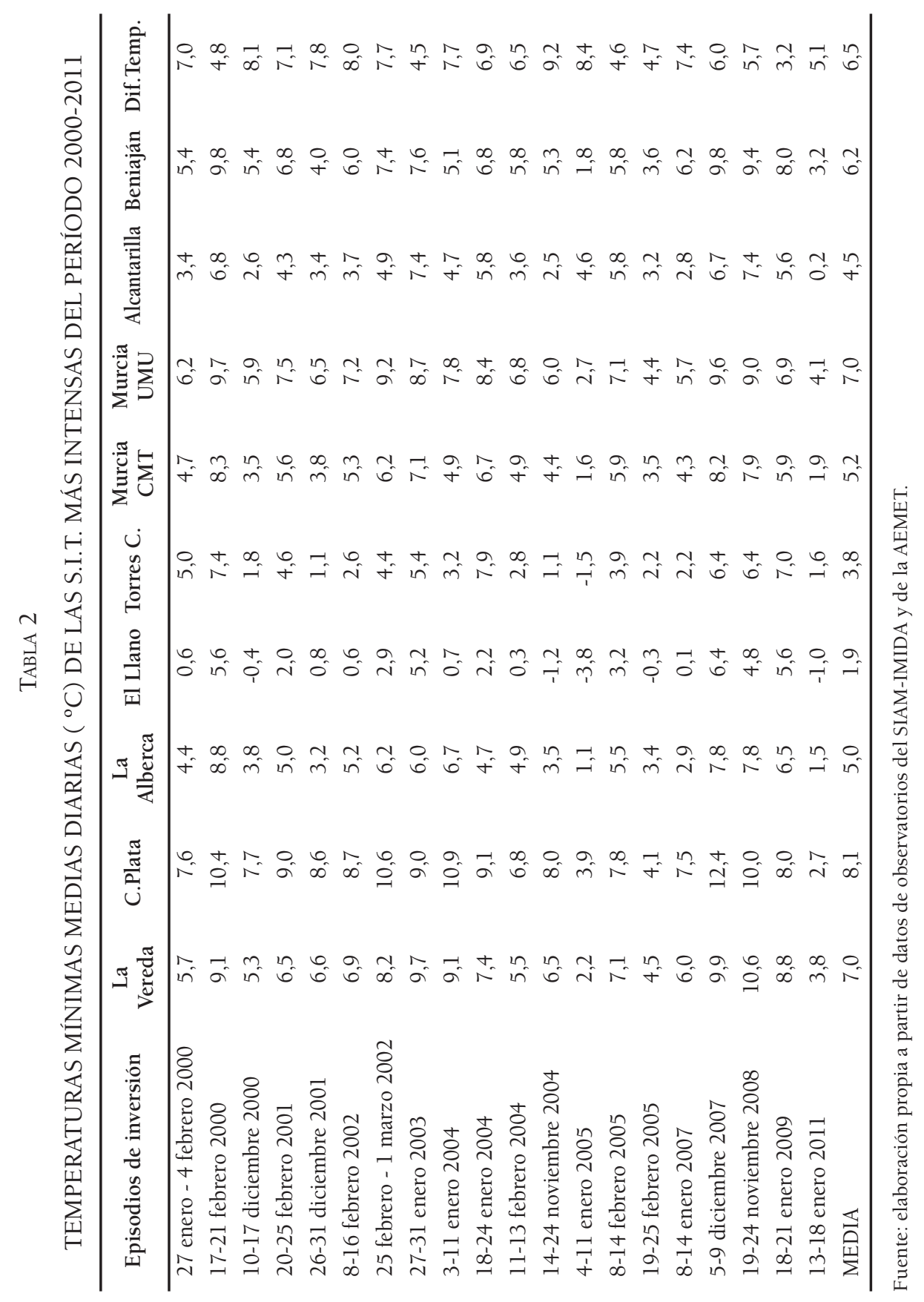

Estudios Geográficos, Vol. LXXV, 277, pp. 521-552, julio-diciembre 2014

ISSN: 0014-1496, eISSN: 1988-8546, doi: 10.3989/estgeogr.201416 


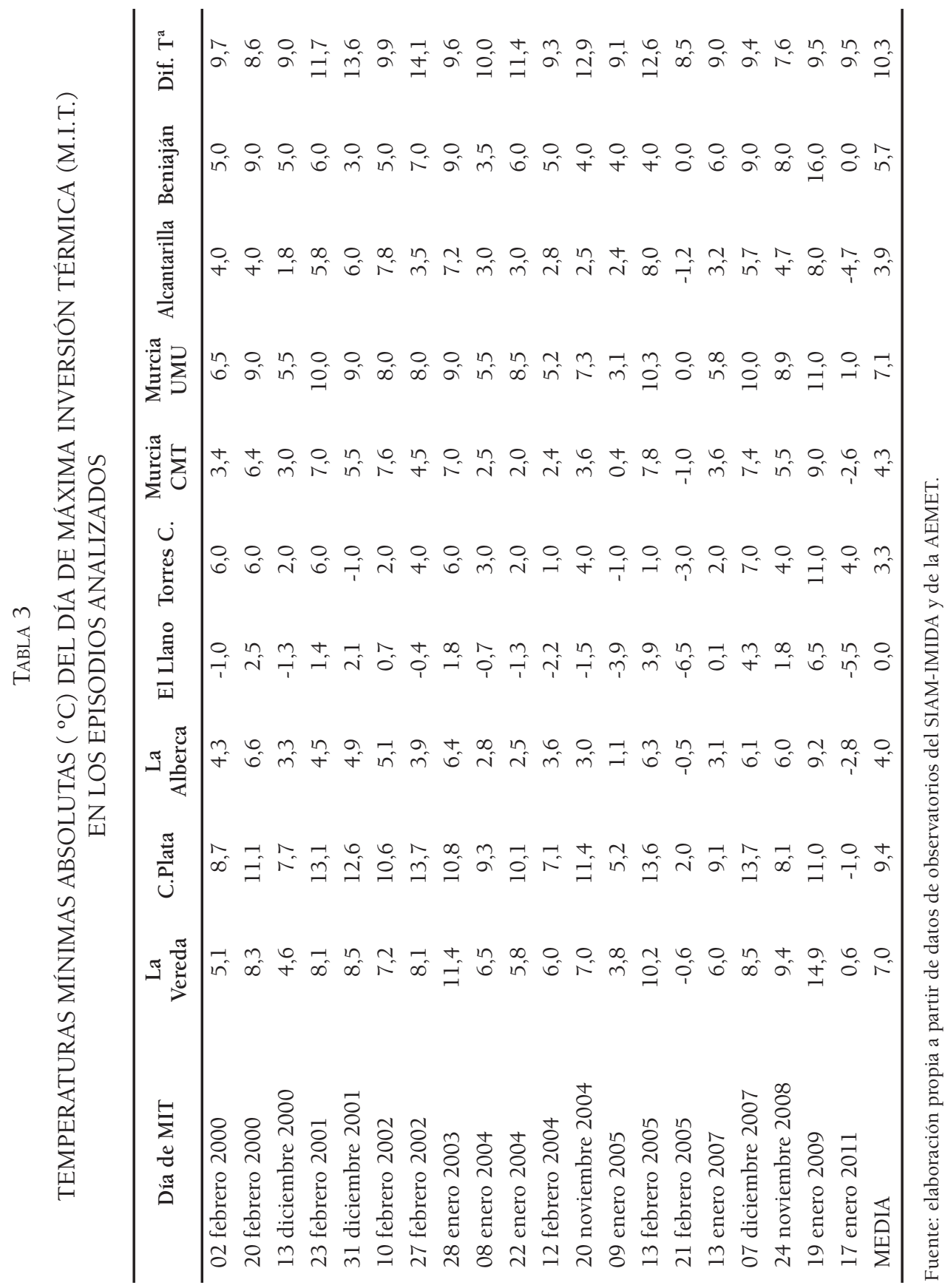




\section{Velocidad y dirección del viento}

La velocidad del viento es, junto con la nubosidad, uno de los factores que más influye en los procesos de inversión térmica (García de Pedraza, 1962). De hecho, cuando un estrato frío superficial por irradiación es alterado por un flujo moderado de viento, con velocidad normalmente superior a los 9,0 $\mathrm{km} / \mathrm{h}$, se favorece la mezcla y la capa más templada superior tiende a descender, rompiendo por tanto el nivel de inversión térmica.

En general, la velocidad media global de las SIT analizadas es muy débil $(3,9 \mathrm{~km} / \mathrm{h})$, siendo el viento en muchos casos prácticamente imperceptible. Las mayores velocidades medias del viento se registran en Cabezo de la Plata $(5,8 \mathrm{~km} / \mathrm{h})$, debido a su configuración topográfica en resalte y mucho más abierta que en la zona de valle. Por el contrario, las velocidades medias más bajas se registran en La Alberca $(2,3 \mathrm{~km} / \mathrm{h})$ y Llano de Molina $(3,5 \mathrm{~km} / \mathrm{h})$ (tabla 4), coincidiendo con una mayor presencia de situaciones de pantano barométrico en superficie. La diferencia media en la velocidad del viento entre los distintos observatorios es de $3,6 \mathrm{~km} / \mathrm{h}$, y solamente en cinco SIT las diferencias de velocidad media superan los $4,0 \mathrm{~km} / \mathrm{h}$.

Los días con mayor inversión térmica dentro de los episodios analizados muestran diferencias en la velocidad del viento algo más acusadas entre las estaciones normalmente expuestas al fenómeno de IT y las marginales $(4,9$ $\mathrm{km} / \mathrm{h}$ como promedio). El contraste más notorio $(19,7 \mathrm{~km} / \mathrm{h}$ de diferencia) corresponde al 24 de enero de 2009, día en el que la velocidad media del viento alcanzada en el Cabezo de la Plata $(27,5 \mathrm{~km} / \mathrm{h})$ fue casi cuatro veces superior a la registrada en La Alberca $(7,8 \mathrm{~km} / \mathrm{h})$. Por otra parte, al analizar las jornadas de MIT con mayor variación espacial de las temperaturas mínimas en superficie (inversiones térmicas más intensas), se comprueba que los valores de velocidad del viento varían muy poco de una estación a otra, por lo general entre 2,5 y $5 \mathrm{~km} / \mathrm{h}$. El rango de velocidades durante dichas IT es por tanto pequeño y comprende valores bajos, especialmente en la zona del valle, donde observatorios como Llano de Molina han llegado a anotar situaciones de relativa calma, con registros de velocidad muy menguada $(<0,7 \mathrm{~km} / \mathrm{h})$.

Tales diferencias de velocidad no se deben sólo a la altitud, sino también al efecto del relieve en relación con la dirección del viento. Durante las SIT analizadas el viento en el área de estudio tiene dos direcciones predominantes: $\mathrm{N}$ y SO (tabla 5), y ello, en relación con la disposición general ESE -ONO de las alineaciones montañosas béticas de la zona, permite distinguir tres sectores de valle con diferente comportamiento. Por un lado, el sector norte de la Vega (Torres de Cotillas- Llano de Molina), cuya configuración orográfica ofrece 


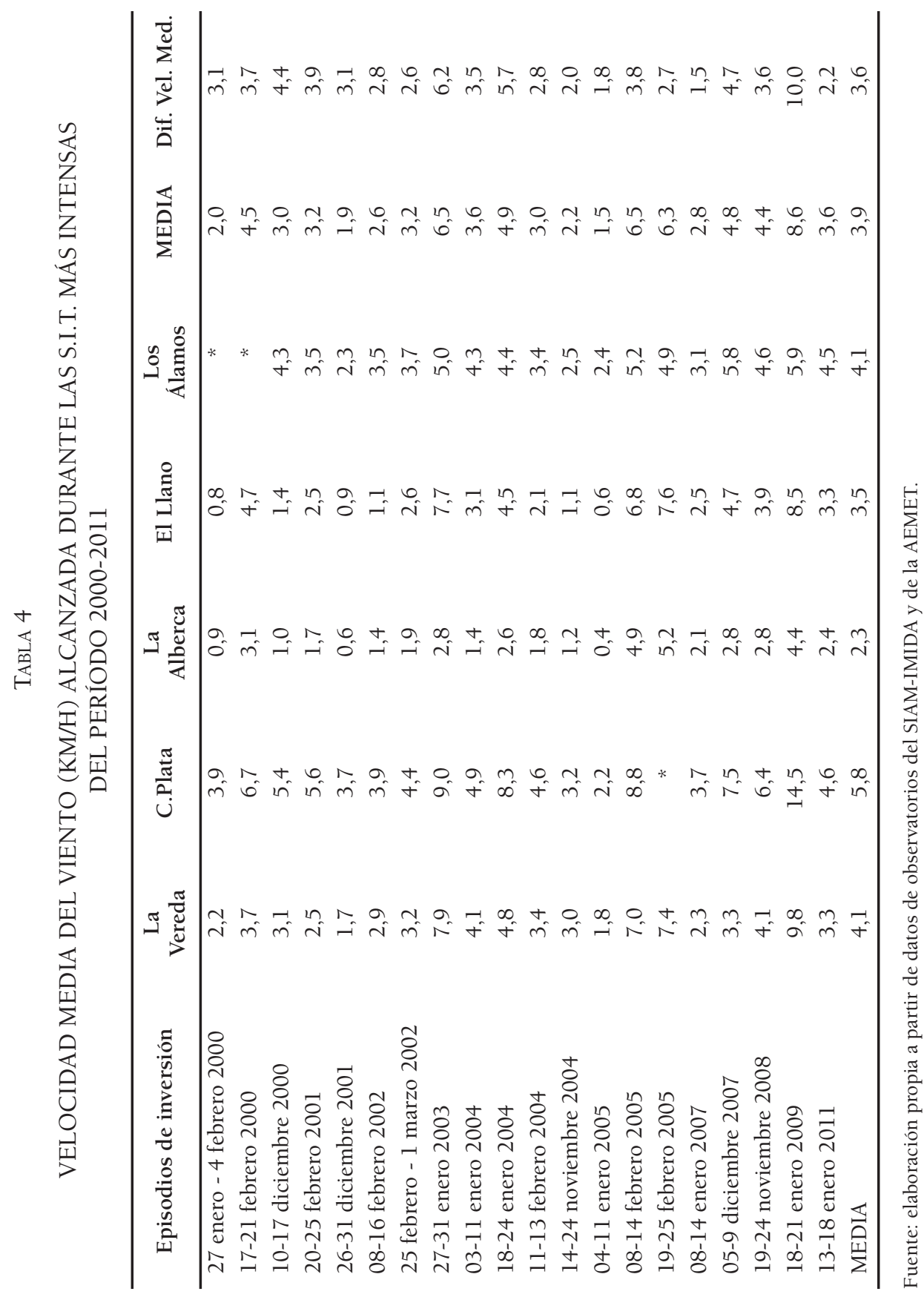


cierto respaldo frente a los vientos de dirección SO, pero no dificulta el paso de los vientos de componente N. En segundo lugar, el sector oeste de la Huerta de Murcia, desde La Contraparada (Alcantarilla) hasta la Alberca, expuesto a ambas direcciones; y por último, el sector oriental de la Huerta de Murcia, desde la ciudad de Murcia y alrededores hasta el límite de la Región de Murcia con la Comunidad Valenciana, abierto totalmente a la influencia de los vientos del SO y algo más protegido respecto a los del $\mathrm{N}$.

En combinación con la reducida carga higrométrica de los niveles medios y bajos atmosféricos, prevalece a menudo una dirección media SO del viento du-

TABLA 5

DIRECCIÓN MEDIA DEL VIENTO DURANTE CADA SITUACIÓN DE INVERSIÓN TÉRMICA ANALIZADA

\begin{tabular}{|c|c|c|c|c|c|c|}
\hline Episodios de inversión & La Vereda & C.Plata & $\begin{array}{c}\text { La } \\
\text { Alberca }\end{array}$ & El Llano & $\begin{array}{c}\text { Los } \\
\text { Álamos } \\
\end{array}$ & MEDIA \\
\hline 27 enero - 4 febrero 2000 & $\mathrm{O}$ & $\mathrm{N}$ & $\mathrm{N}$ & $\mathrm{N}$ & * & $\mathrm{N}$ \\
\hline $17-21$ febrero 2000 & $\mathrm{O}$ & $\mathrm{N}$ & $\mathrm{N}$ & $\mathrm{N}$ & * & $\mathrm{N}$ \\
\hline 10-17 diciembre 2000 & $\mathrm{SO}$ & $\mathrm{N}$ & $\mathrm{N}$ & $\mathrm{N}$ & $\mathrm{SO}$ & $\mathrm{N}$ \\
\hline 20-25 febrero 2001 & SO & $\mathrm{N}$ & $\mathrm{N}$ & $\mathrm{N}$ & $\mathrm{SO}$ & $\mathrm{N}$ \\
\hline 26-31 diciembre 2001 & $\mathrm{O}$ & $\mathrm{N}$ & $\mathrm{N}$ & $\mathrm{N}$ & $\mathrm{SO}$ & $\mathrm{N}$ \\
\hline 08-16 febrero 2002 & $\mathrm{O}$ & $\mathrm{N}$ & $\mathrm{N}$ & $\mathrm{N}$ & $\mathrm{S}$ & $\mathrm{N}$ \\
\hline 25 febrero - 1 marzo 2002 & $\mathrm{SO}$ & $\mathrm{N}$ & $\mathrm{N}$ & * & $\mathrm{SO}$ & $\mathrm{N}$ \\
\hline 27-31 enero 2003 & NO & $\mathrm{N}$ & $\mathrm{N}$ & $\mathrm{N}$ & $\mathrm{SO}$ & $\mathrm{N}$ \\
\hline 03-11 enero 2004 & $\mathrm{SO}$ & $\mathrm{N}$ & $\mathrm{N}$ & $\mathrm{N}$ & $\mathrm{SO}$ & $\mathrm{N}$ \\
\hline 18-24 enero 2004 & * & $\mathrm{N}$ & $\mathrm{N}$ & * & SO & $\mathrm{N}$ \\
\hline 11-13 febrero 2004 & S & $\mathrm{N}$ & $\mathrm{N}$ & $\mathrm{N}$ & $\mathrm{S}$ & $\mathrm{N}$ \\
\hline 14-24 noviembre 2004 & $\mathrm{SO}$ & $\mathrm{N}$ & $\mathrm{N}$ & $\mathrm{N}$ & $S$ & $\mathrm{~N}$ \\
\hline 04-11 enero 2005 & E & $\mathrm{N}$ & $\mathrm{N}$ & $\mathrm{N}$ & $\mathrm{SO}$ & $\mathrm{N}$ \\
\hline 08-14 febrero 2005 & $\mathrm{SO}$ & $\mathrm{N}$ & $\mathrm{N}$ & $\mathrm{N}$ & $\mathrm{SO}$ & $\mathrm{N}$ \\
\hline 19-25 febrero 2005 & NO & $\mathrm{N}$ & $\mathrm{N}$ & $\mathrm{N}$ & $\mathrm{SO}$ & $\mathrm{N}$ \\
\hline 08-14 enero 2007 & SO & * & $S$ & SE & $\mathrm{S}$ & $S$ \\
\hline 05-9 diciembre 2007 & $\mathrm{SO}$ & $\mathrm{N}$ & S & $\mathrm{O}$ & $\mathrm{SO}$ & $\mathrm{SO}$ \\
\hline 19-24 noviembre 2008 & $\mathrm{O}$ & $\mathrm{O}$ & $\mathrm{SO}$ & NO & $\mathrm{SO}$ & $\mathrm{SO}$ \\
\hline 18-21 enero 2009 & $\mathrm{O}$ & $\mathrm{O}$ & $\mathrm{SO}$ & $\mathrm{O}$ & $\mathrm{SO}$ & $\mathrm{O}$ \\
\hline 13-18 enero 2011 & E & SO & S & NO & $\mathrm{SO}$ & $\mathrm{SO}$ \\
\hline MEDIA & $\mathrm{SO}$ & $\mathrm{N}$ & $\mathrm{N}$ & $\mathrm{N}$ & $\mathrm{SO}$ & $\mathrm{N}$ \\
\hline
\end{tabular}

Fuente: elaboración propia a partir de datos de observatorios del SIAM-IMIDA y de la AEMET.

Estudios Geográficos, Vol. LXXV, 277, pp. 521-552, julio-diciembre 2014

ISSN: 0014-1496, eISSN: 1988-8546, doi: 10.3989/estgeogr.201416 
rante las horas nocturnas, o sea un flujo usualmente más cálido y con menor contenido de humedad que la masa de aire a la que sustituye. Esta dirección predomina de forma casi continua en el CMT de Guadalupe (Murcia), aunque varía de forma local en el resto de observatorios. Así, en La Vereda predomina el viento de componente SO, mientras que en los observatorios Cabezo de la Plata, La Alberca y Llano de Molina lo hacen los vientos de dirección N. Teniendo en cuenta este hecho y la influencia orográfica ya descrita, es fácil comprobar la existencia de un patrón que se repite en la mayor parte de las SIT, con idéntica configuración en cinco de ellas y escasas diferencias en el resto (tabla 5).

El predominio de vientos de componente $\mathrm{N}$ en el sector norte de la Vega (Torres de Cotillas - Llano de Molina) explica que el descenso de la temperatura en esta zona de valle sea mayor que en otros sectores topográficamente deprimidos no tan expuestos. La advección de aire algo más frío y pesado tiende a sustituir con relativa rapidez el aire más cálido acumulado durante el día en el fondo de valle, haciendo que se registren aquí los valores de temperatura mínima absoluta más bajos de toda la Vega. Por el contrario, en La Vereda, situada al pie de la Sierra de Carrascoy, predomina el viento de dirección SO, lo que contribuye a un significativo aumento de la temperatura mínima. En el caso de Cabezo de la Plata, al originarse normalmente un estrato muy cálido $\left(15-19^{\circ} \mathrm{C}\right)$ a $200-300 \mathrm{~m}$ de altitud, la masa de aire más frío $(\mathrm{N})$ no puede reemplazarlo, y se desliza en forma de cuña hacia lugares más bajos. No hay que olvidar, y éste es el caso, la influencia que en tales situaciones ejercen los flujos de aire frío catabático (Yao y Zhong, 2009).

\section{Amplitud térmica diaria}

El análisis de la amplitud térmica diaria (ATD) durante las SIT proporciona una información complementaria esencial para determinar la magnitud e intensidad de éstas. Al tratarse de inversiones térmicas en capas bajas de la atmósfera se produce un descenso considerable de los valores mínimos nocturnos, además de un importante ascenso de las temperaturas máximas durante el día. El aire calentado en contacto con el suelo durante el día asciende sólo hasta el nivel de inversión de superficie, conformando una masa cálida de escaso espesor, de temperatura superior a la que existiría en caso de ascenso libre hasta niveles más altos (López Gómez, 1975). De hecho, considerando el total de situaciones, la diferencia máxima de temperatura mínima superficial existente durante la madrugada entre los observatorios del área mantiene una estrecha correlación con la de amplitud térmica diaria (figura 3, A). 
FIGURA 3

(A) DIFERENCIAS MÁXIMAS DE 'ATD’ ENTRE FONDO DE VALLE Y ZONA PERIFÉRICA VERSUS INCREMENTO MÁXIMO DE 'TMS' PARA CADA DÍA DE MIT; (B) INCREMENTO DE TEMPERATURA ENTRE SUPERFICIE Y ESTRATO CÁLIDO VERSUS TEMPERATURA A 850 HPA EN LOS DÍAS DE MÁXIMA INVERSIÓN TÉRMICA
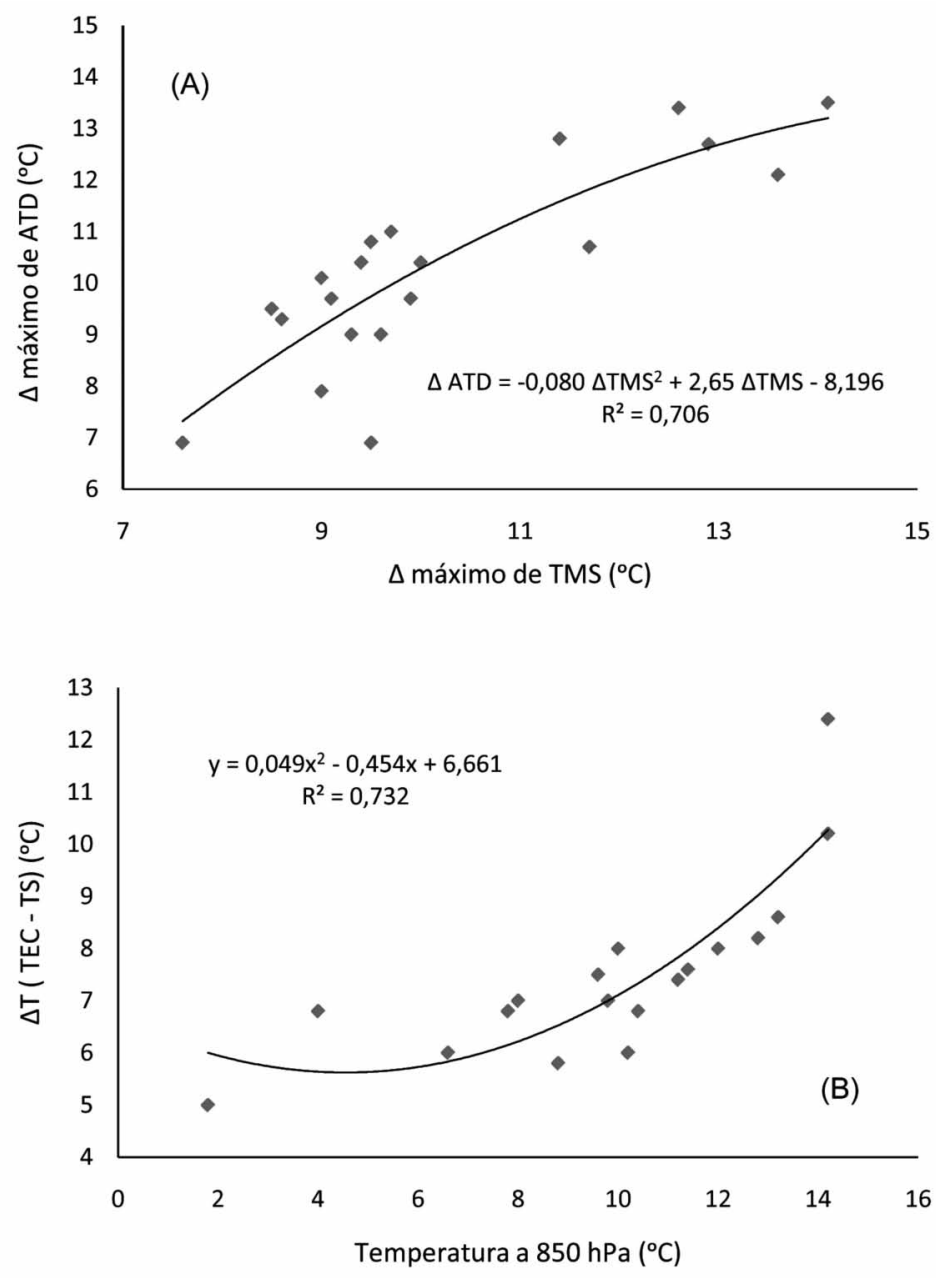

Fuente: elaboración propia a partir de datos suministrados por el SIAM-IMIDA y la AEMET (A) y de los datos de sondeos aerológicos realizados por el Centro Meteorológico Territorial (CMT) de Murcia (B). ATD $=$ amplitud térmica diaria $\left({ }^{\circ} \mathrm{C}\right) ; \mathrm{TMS}=$ temperatura mínima superficial $\left({ }^{\circ} \mathrm{C}\right) ; \Delta \mathrm{T}(\mathrm{TEC}-\mathrm{TS})=$ incremento de temperatura $\left({ }^{\circ} \mathrm{C}\right)$ entre superficie y estrato cálido. 


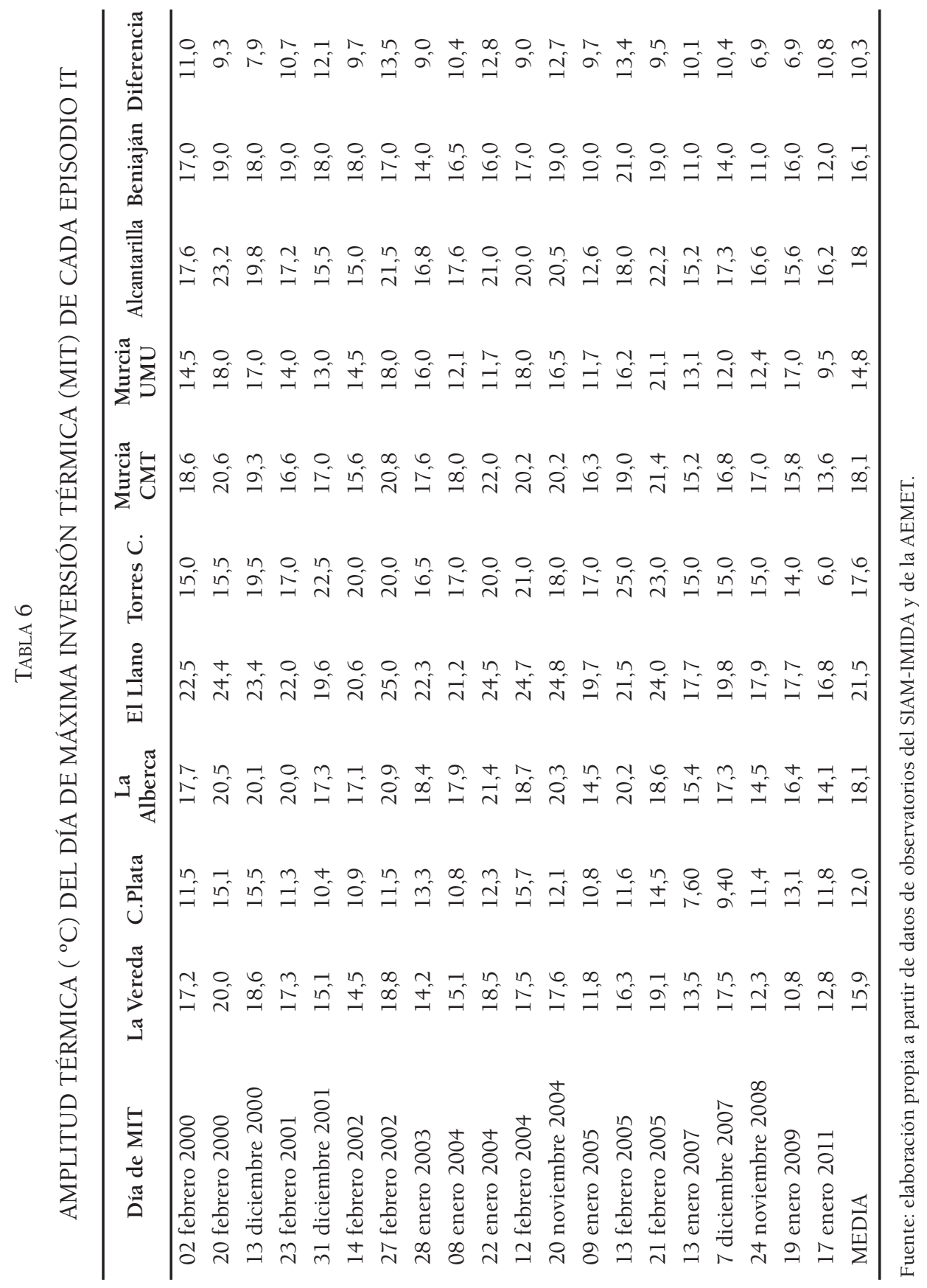


La amplitud térmica diaria media (ATDM) de las estaciones del área durante las SIT es de $13,9^{\circ} \mathrm{C}, 1,5^{\circ} \mathrm{C}$ más que la media invernal estimada para los últimos 30 años $\left(12,4^{\circ} \mathrm{C}\right)$. Como es de esperar, las mayores amplitudes térmicas medias se registran en las zonas de valle: $17,1^{\circ} \mathrm{C}$ en Llano de Molina, $14,7{ }^{\circ} \mathrm{C}$ en Alcantarilla y Torres de Cotillas. Por el contrario, las estaciones localizadas en sectores marginales o más elevados muestran amplitudes térmicas menores, por debajo de la media de invierno (Cabezo de la Plata, $10,9^{\circ} \mathrm{C}$; Murcia UMU, $12,3^{\circ} \mathrm{C}$ ) o en torno a ella (Beniaján, $12,7^{\circ} \mathrm{C}$ ). Entre el fondo del valle y la zona no afectada por la IT se observa, por tanto, una diferencia de ATDM de $6,2^{\circ} \mathrm{C}$, y diferencias de ATD iguales o superiores a $7,0^{\circ} \mathrm{C}$ en el $50 \%$ de las situaciones.

Este contraste entre las tierras bajas de la Vega y los relieves circundantes resulta especialmente notorio en las jornadas de mayor amplitud térmica (tabla 6) dentro de las SIT. En este caso, la diferencia media entre los sectores de ATD máxima (fondo del valle) y mínima (zona periférica), se sitúa en $10,3{ }^{\circ} \mathrm{C}$, umbral, por otra parte, superado en once de las 20 jornadas MIT de los episodios de inversión térmica analizados ( $55 \%$ de las SIT). El mayor contraste entre ambas zonas (diferencia de ATD de $13,5^{\circ} \mathrm{C}$ ), asociado a una fuerte intensificación del fenómeno de inversión térmica, corresponde al 27 de febrero de 2002, fecha en que el Llano de Molina alcanzó los $25,0{ }^{\circ} \mathrm{C}$ de ATD, frente a los $11,5^{\circ} \mathrm{C}$ del Cabezo de la Plata. Ello se debió a un intenso enfriamiento nocturno mucho más acusado en El Llano que en los terrenos altos, frente a valores máximos de temperatura relativamente homogéneos en toda el área.

\section{Datos extraídos de sondeos aerológicos}

Los sondeos aerológicos del CMT de Guadalupe (Murcia), tomados a medianoche (00Z), arrojan resultados de gran interés. Cabe señalar, en primer lugar, que las temperaturas registradas en superficie a dicha hora durante cada una de las situaciones de inversión térmica no son excesivamente bajas. La media resultante es de $9,4^{\circ} \mathrm{C}$, lo que implica la existencia de períodos relativamente poco fríos, con valores por encima de lo habitual en la estación invernal. Considerando el conjunto de SIT, el estrato cálido ofrece una temperatura media de $12,7^{\circ} \mathrm{C}$, o sea $4,3^{\circ} \mathrm{C}$ más que en superficie. Dicho contraste es mucho más acusado en determinadas SIT, como las del 10-17 de diciembre de 2000 y 26-31 de diciembre de 2001, en las que se aprecian incrementos medios superiores a $8{ }^{\circ} \mathrm{C}$ entre ambos niveles. El patrón de temperatura media en el geopotencial de $850 \mathrm{hPa}$ es bastante significativo, ya que los valores térmicos a esta altitud, 1.500 metros aproximadamente, adquieren una importante 
anomalía positiva en relación con la media habitual de invierno $\left(7,1^{\circ} \mathrm{C}\right.$ frente a los $4,0^{\circ} \mathrm{C}$ de media invernal en los últimos 30 años). De hecho, cuanto más alta es la temperatura a $850 \mathrm{hPa}$ mayor es la diferencia térmica entre la superficie y el estrato cálido (figura 3.B).

TABLA 7

DATOS DE SONDEOS AEROLÓGICOS DE LOS DÍAS CON MAYOR CONTRASTE TÉRMICO ENTRE SUPERFICIE Y ESTRATO CÁLIDO (00 H, CMT MURCIA)

\begin{tabular}{|c|c|c|c|c|c|c|c|}
\hline Día de MIT & $\mathrm{TS}\left({ }^{\circ} \mathrm{C}\right)$ & $\operatorname{TEC}\left({ }^{\circ} \mathrm{C}\right)$ & $\begin{array}{r}\mathrm{AEC} \\
(\mathrm{m})\end{array}$ & $\begin{array}{c}\text { Ratio } \\
{ }^{\circ} \mathrm{C} / 100 \mathrm{~m} .\end{array}$ & $\begin{array}{l}\Delta \mathrm{T}(\mathrm{TEC}- \\
\mathrm{TS})\left({ }^{\circ} \mathrm{C}\right)\end{array}$ & $\begin{array}{c}\mathrm{T} 850 \\
\mathrm{hPa}\left({ }^{\circ} \mathrm{C}\right)\end{array}$ & $\begin{array}{c}\text { Viento } \\
10 \mathrm{~m}\end{array}$ \\
\hline 02 febrero 2000 & 20,6 & 11,8 & 243 & 3,6 & 8,8 & 9 & $\mathrm{OSO}$ \\
\hline 20 febrero 2000 & 13 & 20,4 & 241 & 3,1 & 7,4 & 11,2 & $\mathrm{SO}$ \\
\hline 13 diciembre 2000 & 7,2 & 17,4 & 921 & 1,1 & 10,2 & 14,2 & $\mathrm{NO}$ \\
\hline 23 febrero 2001 & 9,6 & 17,6 & 277 & 2,9 & 8 & 10 & $\mathrm{SO}$ \\
\hline 31 diciembre 2001 & 6 & 18,4 & 662 & 1,9 & 12,4 & 14,2 & OSO \\
\hline 14 febrero 2002 & 9,4 & 17,6 & 729 & 1,1 & 8,2 & 12,8 & OSO \\
\hline 27 febrero 2002 & 11,2 & 19,8 & 296 & 2,9 & 8,6 & 13,2 & SO \\
\hline 28 enero 2003 & 10 & 17 & 498 & 1,4 & 7 & 9,8 & NO \\
\hline 08 enero 2004 & 8,2 & 14,2 & 224 & 2,7 & 6 & 6,6 & SOS \\
\hline 22 enero 2004 & 6,6 & 14,6 & 458 & 1,7 & 8 & 6,2 & $\mathrm{SO}$ \\
\hline 12 febrero 2004 & 7,6 & 14,6 & 685 & 1 & 7 & 8 & $\mathrm{O}$ \\
\hline 20 noviembre 2004 & 9 & 16,6 & 351 & 2,2 & 7,6 & 11,4 & $\mathrm{O}$ \\
\hline 09 enero 2005 & 2,8 & 10,8 & 977 & 0,8 & 8 & 6,8 & SSE \\
\hline 13 febrero 2005 & 10,6 & 16,6 & 216 & 2,8 & 6 & 10,2 & $\mathrm{SO}$ \\
\hline 21 febrero 2005 & 6,8 & 11,8 & 343 & 1,5 & 5 & 1,8 & $\mathrm{SO}$ \\
\hline 13 enero 2007 & 6,4 & 14,4 & 1106 & 0,7 & 8 & 12 & $\mathrm{SO}$ \\
\hline 7 diciembre 2007 & 9,4 & 16,2 & 261 & 2,6 & 6,8 & 10,4 & $\mathrm{O}$ \\
\hline 24 noviembre 2008 & 9,2 & 16 & 172 & 4 & 6,8 & 7,8 & $\mathrm{NO}$ \\
\hline 19 enero 2009 & 9,4 & 13,8 & 264 & 1,7 & 4,4 & 8,4 & $\mathrm{O}$ \\
\hline 17 enero 2011 & 7 & 12,8 & 927 & 0,6 & 5,8 & 8,8 & $\mathrm{SO}$ \\
\hline MEDIA & 8,6 & 16,1 & 492,6 & 2 & 7,5 & 9,6 & $\mathrm{SO}$ \\
\hline
\end{tabular}

TS $=$ Temperatura en superficie $; \mathrm{TEC}=$ Temperatura del estrato cálido; $\mathrm{AEC}=$ Altitud del estrato cálido; $\Delta \mathrm{T}$ $($ TEC-TS $)$ = Incremento de temperatura entre superficie y estrato cálido.

Fuente: elaboración propia a partir de datos de sondeos aerológicos realizados por el Centro Meteorológico Territorial (CMT) de Murcia. 


\section{FIGURA 4}

\section{COMPARACIÓN ENTRE GRADIENTE TÉRMICO, INCREMENTO \\ DE TEMPERATURA DESDE LA SUPERFICIE AL ESTRATO CÁLIDO Y ALTITUD \\ DEL EC EN LOS DÍAS DE M.I.T. DE LAS S.I.T. ANALIZADAS}

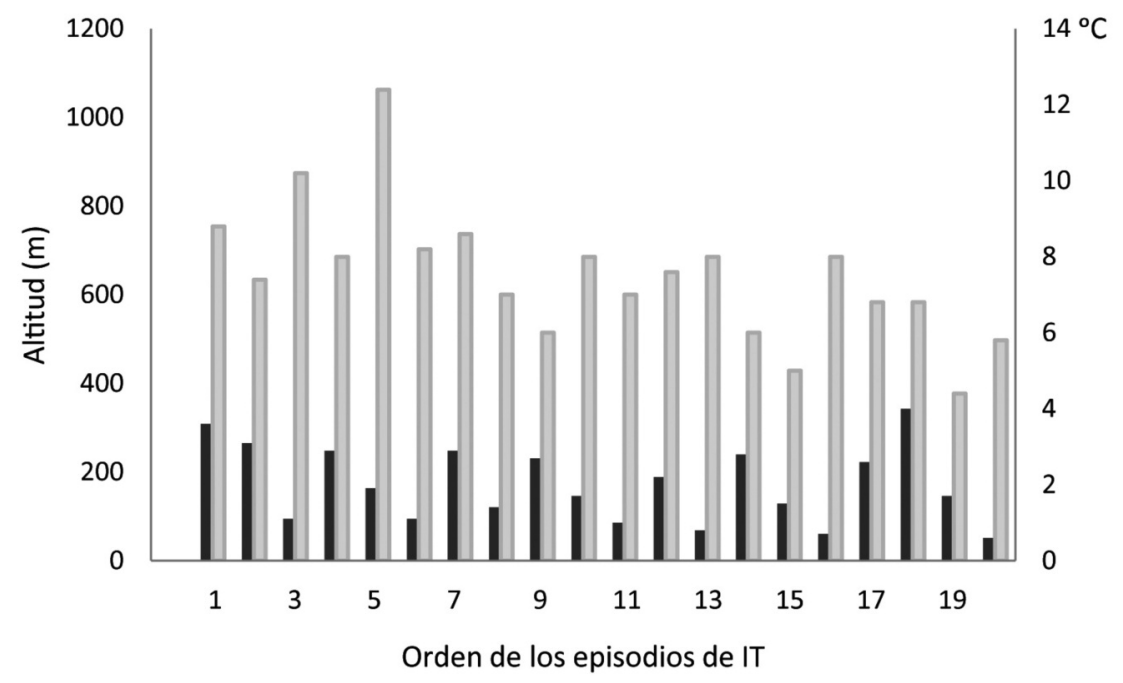

Ratio ${ }^{\circ} \mathrm{C} / 100 \mathrm{~m}$

$\Delta \mathrm{T}(\mathrm{TEC}-\mathrm{TS})$

- Altitud EC (m)

TS = Temperatura en superficie $\left({ }^{\circ} \mathrm{C}\right) ;$ TEC $=$ Temperatura del estrato cálido $\left({ }^{\circ} \mathrm{C}\right) ;$ AEC $=$ Altitud del estrato cálido $(\mathrm{m}) ; \Delta \mathrm{T}(\mathrm{TEC}-\mathrm{TS})=$ Incremento de temperatura $\left({ }^{\circ} \mathrm{C}\right)$ entre superficie y estrato cálido.

Fuente: elaboración propia a partir de datos de sondeos aerológicos realizados por el Centro Meteorológico Territorial (CMT) de Murcia.

De la información suministrada por los sondeos se infiere que, durante las jornadas de máxima inversión térmica (MIT), el estrato cálido se sitúa a una altitud media de 492,5 $\mathrm{m}(964,7 \mathrm{hPa})$ y la ratio de descenso medio de temperatura desde dicho estrato hasta la superficie en torno a $-2{ }^{\circ} \mathrm{C} / 100 \mathrm{~m}$ (tabla 7 ). En situaciones extremas, esta ratio representa descensos térmicos muy bruscos (figura 4); tal es el caso de las inversiones de temperatura producidas el 2 de febrero de 2000 y el 24 de noviembre de 2008 durante las que se alcanzaron ratios de $-3,5^{\circ} \mathrm{C}$ y de $-4{ }^{\circ} \mathrm{C} / 100 \mathrm{~m}$ respectivamente. Asimismo, se observa un significativo incremento de las diferencias térmicas entre el nivel de superficie y de altura, con respecto a los promedios hallados para los episodios completos de inversión. La temperatura superficial $\left(8,6^{\circ} \mathrm{C}\right.$ de media para el total de días de MIT) experimenta poca variación en relación con el promedio 
estimado para las SIT, apenas $0,8^{\circ} \mathrm{C}$ menos. En cambio, los valores medios de temperatura del estrato cálido y de la topografía de $850 \mathrm{hPa}$ calculados en los días de MIT, $16,1^{\circ} \mathrm{C}$ y $9,6^{\circ} \mathrm{C}$ respectivamente, sí suponen incrementos apreciables respecto a las medias de los episodios. Este hecho parece estar relacionado con la mayor proximidad de la dorsal africana durante los días de máxima inversión térmica, y por consiguiente con el desarrollo de una más intensa advección cálida en niveles medios y bajos atmosféricos.

\section{ANÁLISIS SINÓPTICO DE LAS SITUACIONES DE INVERSIÓN TÉRMICA}

\section{Situación de inversión térmica 20-25 de febrero de 2001}

Durante los días 21 y 22 de febrero de 2001 predomina en Europa occidental una circulación meridiana de alto grado que genera una situación de bloqueo con disposición en «Omega». Dicho bloqueo es producido por una enérgica célula anticiclónica cálida en latitudes altas localizada al oeste del Canal de la Mancha $(1.035 \mathrm{hPa})$. La dorsal cálida, con eje oblicuo SO-NE, es alimentada por aire subtropical de bajas latitudes, «inmovilizado en latitudes septentrionales por los chorros que lo limitan al oeste y al este» (Capel Molina, 2001).

En altura, existen dos Depresiones Aisladas en Niveles Altos (DANAs), una de ellas, con centro depresionario al suroeste del Cabo San Vicente, reflejada en superficie por una perturbación atlántica y un frente ocluido asociado. En superficie, la Península Ibérica queda bajo el dominio de las altas presiones $(1.025 \mathrm{hPa})$. En la topografía de $850 \mathrm{hPa}$ se advierte una irrupción cálida del $\mathrm{SO}$, cuya cresta dorsalina alcanzaba la vertical del noroeste español con temperaturas entre 8 y $11{ }^{\circ} \mathrm{C}$.

A medida que avanzan las horas, el vórtice frío en niveles altos se reintegra a la Circulación General del Oeste (CGO), provocando la continuación del flujo del SO en superficie sobre el tercio suroriental peninsular, y haciendo aún más patente la advección de origen subtropical sobre la Región de Murcia. Comienzan así a contabilizarse las primeras inversiones absolutas nocturnas, con temperaturas mínimas el día $22\left(-1^{\circ} \mathrm{C}\right)$ en El Llano.

Durante el día 23 de febrero de 2001, jornada de máxima inversión térmica, se debilita el Anticiclón de las Azores, aunque la Península Ibérica continúa todavía bajo influencia anticiclónica $(1.015 \mathrm{hPa})$. En el Sureste peninsular se advierte un claro pantano barométrico, con viento prácticamente inapreciable $(2,9 \mathrm{~km} / \mathrm{h})$. En la superficie de $850 \mathrm{hPa}$ se reforzaron la transgresión de aire subtropical marítimo y la dorsal cálida con temperaturas de hasta $11{ }^{\circ} \mathrm{C}$ (figura 5). 
FIGURA 5

MAPA SINÓPTICO EN SUPERFICIE (1), GEOPOTENCIAL Y TEMPERATURA A 850 HPA (2) (23/02/2001; 00Z)
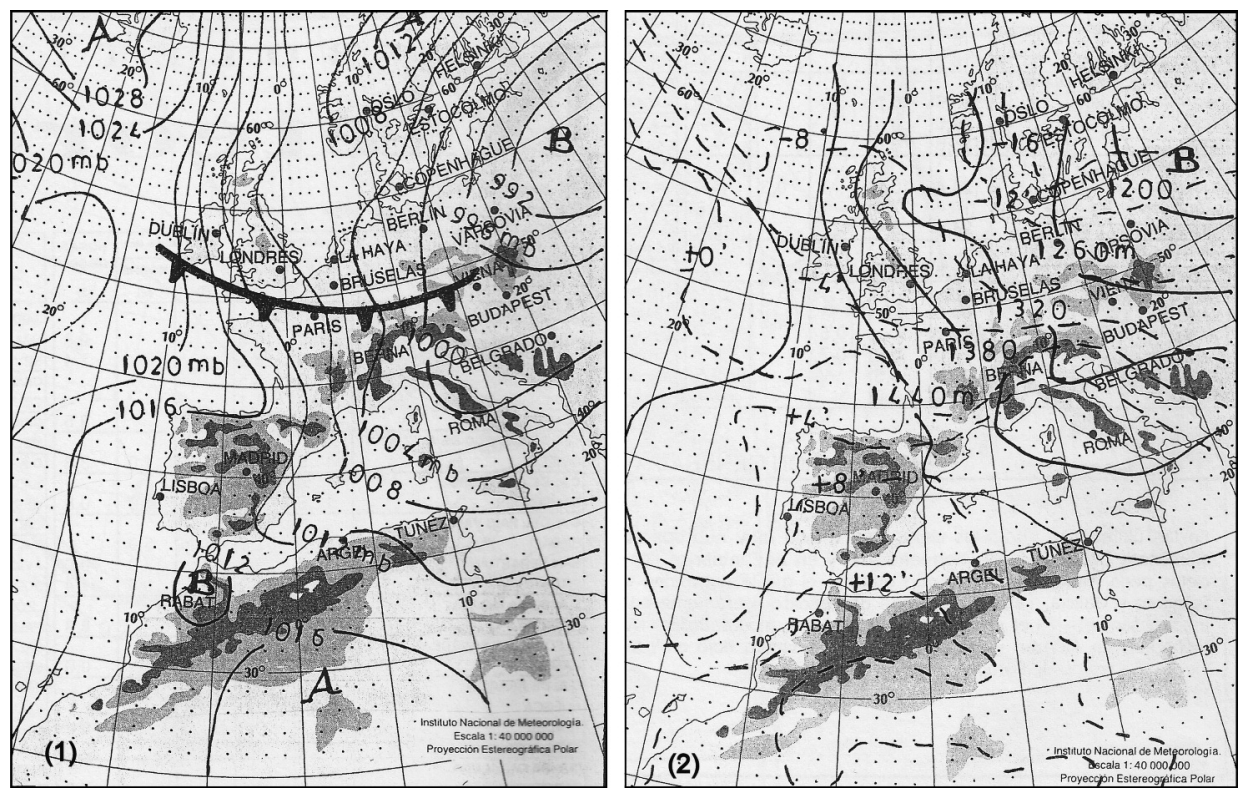

Fuente: Boletín Meteorológico del Instituto Nacional de Meteorología (INM).

Esta situación sinóptica general se tradujo en la Vega Media del Segura en una jornada estable con cielo despejado, como reflejan las más de 10 horas de sol que se contabilizaron en el observatorio de Guadalupe (Murcia). La amplitud térmica media, por consiguiente, fue muy elevada $\left(20,4^{\circ} \mathrm{C}\right)$, especialmente en las zonas de valle, donde se rebasaron los $24^{\circ} \mathrm{C}: 25,5^{\circ} \mathrm{C}$ en Llano de Molina frente a los $16,8{ }^{\circ} \mathrm{C}$ del Cabezo de la Plata. La disparidad de casi $9{ }^{\circ} \mathrm{C}$ entre ambas estaciones se debió a sus diferentes valores de temperatura mínima, más que a las máximas absolutas registradas, bastante similares $\left(23,4{ }^{\circ} \mathrm{C}\right.$ y $24,4^{\circ} \mathrm{C}$ respectivamente). La inversión térmica absoluta llegó a su cénit durante dicho día, con un marcado descenso de la temperatura mínima de superficie en el fondo del valle $\left(1,4^{\circ} \mathrm{C}\right.$ en Llano de Molina) respecto a la registrada en terrenos altos marginales $\left(13,1^{\circ} \mathrm{C}\right.$ en Cabezo de la Plata). Según datos del sondeo atmosférico del CMT de Guadalupe, a las 00Z existía un estrato muy cálido $\left(17,6^{\circ} \mathrm{C}\right)$ a $985 \mathrm{hPa}(277$ metros de altitud), mientras que en superficie la temperatura descendió hasta los 9,6 ${ }^{\circ} \mathrm{C}$. La diferencia en atmós- 
fera libre fue, por tanto, de $8{ }^{\circ} \mathrm{C}$, siendo llamativo que tal contraste pudiera producirse en menos de $300 \mathrm{~m}$.

Durante los días 24 y 25 de febrero, la Península Ibérica va quedando bajo el dominio de las bajas presiones $(1.010 \mathrm{hPa})$. Una potente perturbación centroeuropea, con sistema frontal de carácter frío asociado, generó inestabilidad en la mitad norte peninsular. En el Sureste peninsular las lluvias se debieron a la reinserción de la DANA a la CGO, cuya zona de divergencia en niveles medios-altos de la atmósfera se situó sobre la vertical de la Región de Murcia. En la superficie de $850 \mathrm{hPa}$ se produjo el debilitamiento de la dorsal cálida argelina, que, con un claro descenso de temperatura, fue progresivamente sustituida por una masa de aire frío polar marítimo.

\section{Situación de inversión térmica 25-29 de febrero de 2002}

Durante las jornadas 25 y 26 de febrero de 2002 se advierte en Europa occidental, a nivel de superficie, una marcada circulación zonal del Oeste, con un centro de acción anticiclónico de bloqueo -Anticiclón de las Azores (1.025 hPa) - que deja a la Península Ibérica bajo el dominio de las altas presiones $(1.020 \mathrm{hPa})$ y a la mitad meridional (Andalucía, La Mancha y Levante) en situación de pantano barométrico. En niveles altos de la atmósfera se produce una advección del NO con una dorsal de onda muy larga al SO de la Península Ibérica, alimentada en el geopotencial de $850 \mathrm{hPa}$ por una burbuja relativamente cálida para esta época del año $\left(12^{\circ} \mathrm{C}\right)$, que se extiende sobre la mitad sur peninsular.

Con el paso de las horas, las altas presiones $(1.020 \mathrm{hPa})$ continuaron asentadas en la mitad meridional peninsular, aunque con un importante y apretado haz de isobaras paralelas a las isohipsas en la cornisa cantábrica; todo ello unido a la entrada de un sistema frontal frío asociado a bajas presiones con dos claros centros ciclónicos sobre el Mar Báltico (972 hPa). En capas altas de la atmósfera se desarrolla una circulación neta del SO que acarrea la llegada de una masa cálida de origen subtropical marítimo, inicialmente vinculada a la masa descendente del anticiclón subtropical de Azores (Capel Molina, 2001).

La presencia de la gran burbuja cálida del SO Peninsular se manifiesta el día 26 sobre la Región de Murcia mediante una fuerte anomalía térmica positiva con isotermas de 12 y $13^{\circ} \mathrm{C}$ entre 1.550 y $1.560 \mathrm{~m}$ de altitud. Esta situación sinóptica genera inversiones térmicas significativas, con temperaturas mínimas absolutas muy contrastadas, de $11^{\circ} \mathrm{C}$ en Cabezo de la Plata y $1{ }^{\circ} \mathrm{C}$ en Llano de Molina.

Durante el 27 de febrero, jornada de máxima inversión térmica, el sur de España sigue bajo la influencia de las altas presiones (1.020 hPa), mientras que el cuadrante noroccidental sufre el avance del sistema frontal frío asociado al 
complejo de bajas presiones de Escandinavia ( 970 y $975 \mathrm{hPa})$. En el área de estudio persiste la situación de pantano barométrico con viento prácticamente nulo $(2,7 \mathrm{~km} / \mathrm{h})$. En niveles altos, un claro flujo subtropical marítimo provoca la irrupción de una masa de aire cálido del Suroeste, cuya cresta dorsalina se inclina hacia el ENE, situándose sobre el Sureste peninsular. De hecho, en el geopotencial de los $850 \mathrm{hPa}$ sobre la Región de Murcia, a las 00Z, aparece trazada la isoterma de $14{ }^{\circ} \mathrm{C}$ (figura 6). Como consecuencia, la Vega Media del Segura disfrutó de un día soleado (casi 9 horas de sol en la capital murciana) (CMT de Guadalupe) y registró valores de amplitud térmica diaria muy altos $\left(25,8^{\circ} \mathrm{C}\right.$ en El Llano de Molina y $21,5^{\circ} \mathrm{C}$ en Alcantarilla). Los datos del sondeo atmosférico del CMT de Guadalupe, a las 00Z, corroboran que existía un estrato extraordinariamente cálido, de $19,8^{\circ} \mathrm{C}$ a $985 \mathrm{hPa}(296 \mathrm{~m})$, mientras que, a nivel superficial, la temperatura descendió hasta los $11,2^{\circ} \mathrm{C}$. En la atmósfera libre, la diferencia térmica entre ambas capas fue de $8,6^{\circ} \mathrm{C}$, evidenciándose en el Diagrama de Stüve una fuerte inversión térmica de irradiación, con aumento súbito de la humedad relativa (HR) en superficie (71\%), frente a la contenida en el estrato cálido a $985 \mathrm{hPa}(19 \%)$. Asimismo, se produjeron diferencias de temperatura muy notorias entre los observatorios del valle, con helada nocturna y mínima absoluta de $-0,4^{\circ} \mathrm{C}$ en Llano de Molina, y los situados fuera del área de IT, con mínimas superiores a los $13^{\circ} \mathrm{C}\left(13,7^{\circ} \mathrm{C}\right.$ en Cabezo de la Plata).

Progresivamente, entre los días 28 de febrero y 1 de marzo, las altas presiones sobre la Península Ibérica se fueron debilitando a medida que el Anticiclón de las Azores se desplazaba hacia latitudes septentrionales, fusionándose con el Anticiclón Groenlandés. Las bajas presiones relativas (1.004 hPa) comenzaron a invadir el tercio noroccidental peninsular con la entrada de un sistema frontal ocluido asociado a la zona divergente de una gran vaguada polar de onda muy larga. En altura, una masa de aire polar marítimo entró por el N de la Península, con clara advección zonal del Oeste. Paulatinamente se produjo el desplazamiento de la cresta de la dorsal argelina hacia el Mediterráneo central. A medida que pasaban las horas, la inestabilidad atmosférica fue haciéndose patente en la mitad oriental española, cuyo frente frío invadió la Región de Murcia, dejando $12 \mathrm{~mm}$ de precipitación el 1 de marzo. En capas altas se manifestó la expansión de la masa polar marítima sobre la totalidad de la Península Ibérica, incorporando mayor nubosidad y anulando por completo la inversión térmica descrita.

\section{Situación de inversión térmica 4-11 de enero de 2005}

El día 7 de enero de 2005, predominaba en Europa occidental una circulación zonal del Oeste con escaso gradiente barométrico sobre la Península Ibé- 


\section{FIGURA 6}

\section{MAPA SINÓPTICO EN SUPERFICIE (1), GEOPOTENCIAL Y TEMPERATURA A 850 HPA (2) $(27 / 02 / 2002 ; 00 Z)$}
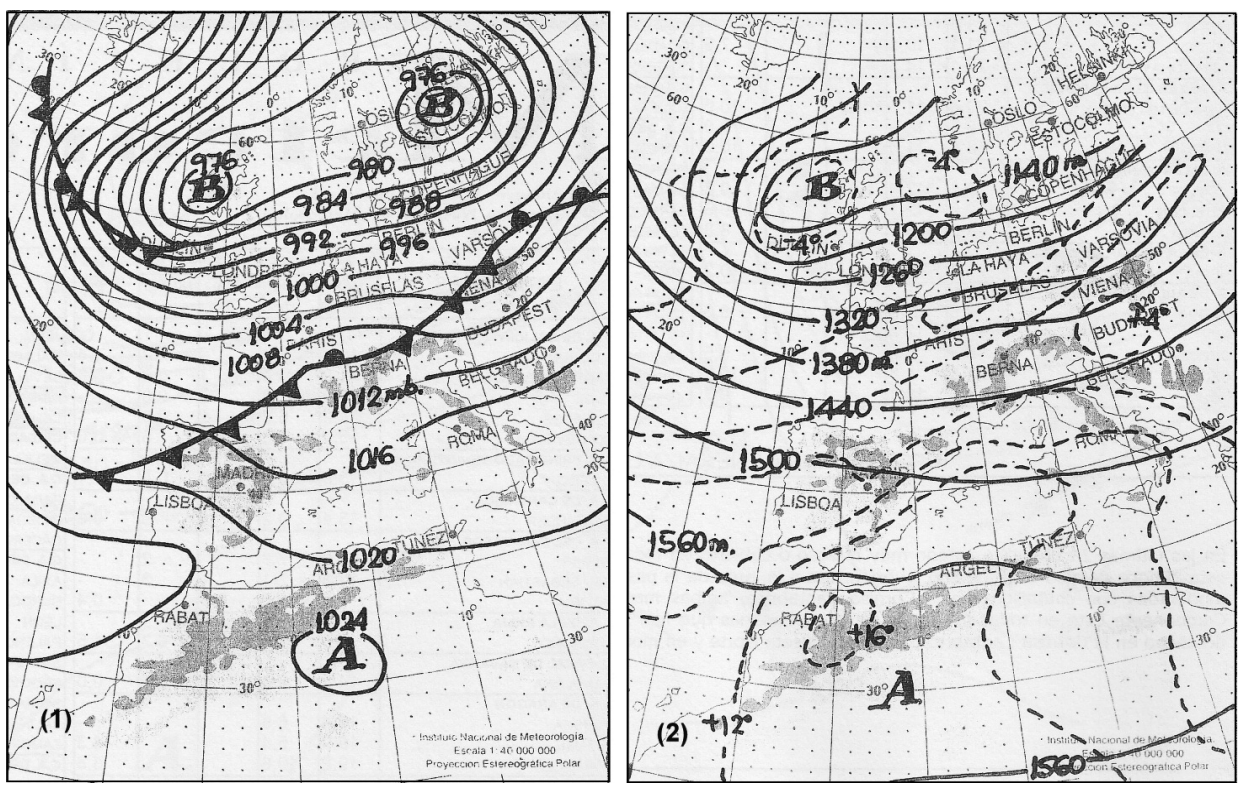

Fuente: Boletín Meteorológico del Instituto Nacional de Meteorología (INM).

rica. El territorio peninsular quedaba así bajo el dominio de las altas presiones subtropicales y la influencia del Anticiclón Europeo (1.040 hPa sobre la mitad norte peninsular), convertido en un gran pantano barométrico. En altura, destacaba la presencia de una potente burbuja cálida aislada sobre la vertical del solar ibérico, junto a una débil advección del NO.

El gran Anticiclón europeo permaneció varias horas con presiones muy elevadas en su centro $(1.035 \mathrm{hPa})$ y dos dorsales de escasa longitud de onda: una próxima a Madeira y otra que alcanzaba el Mar Negro. Un sistema frontal frío asociado a una importante perturbación (968 hPa), posicionada sobre el Sur de Noruega, avanzó hacia el NO peninsular, dificultando las altas presiones reinantes en latitudes más meridionales. En capas altas de la atmósfera seguía predominando un flujo débil del Oeste, que marcaba $10{ }^{\circ} \mathrm{C}$ en el geopotencial de $850 \mathrm{hPa}$ sobre la fachada mediterránea española.

En la jornada de máxima inversión térmica, 9 de enero de 2005, continuaba la estabilidad atmosférica, con un acusado máximo anticiclónico central 
$(>1.035 \mathrm{hPa}$ ) (figura 7). Las altas presiones se afianzaron creando una situación de bloqueo ante las perturbaciones atlánticas de latitudes septentrionales (mínimo de $976 \mathrm{hPa}$ al NO de Irlanda). La circulación zonal del Oeste persistía también en los niveles medios y altos de la atmósfera. Como resultado, la dorsal cálida, que días anteriores afectaba al litoral mediterráneo, fue desplazándose hacia el Este, provocando un claro descenso de la temperatura en el geopotencial de $850 \mathrm{hPa}\left(7^{\circ} \mathrm{C}\right)$. Durante esta jornada, el tiempo fue estable y soleado en toda la Vega Media del Segura (9,3 horas de sol según el CMT de Guadalupe). La diferencia térmica entre la temperatura real a $500 \mathrm{hPa}$ y la parcela de aire adiabática que ascendía desde el nivel de $850 \mathrm{hPa}$ hasta su nivel de condensación fue muy acusada, eliminando cualquier tipo de inestabilidad en capas bajas atmosféricas (Showalter, 1953). Además, el escaso gradiente barométrico propició una jornada con viento prácticamente imperceptible (1,3 $\mathrm{km} / \mathrm{h}$ ). A pesar del ambiente estable, escaso viento y numerosas horas de sol, la amplitud térmica media no fue tan elevada, debido al registro de temperaturas

\section{FIGURA 7}

\section{MAPA SINÓPTICO EN SUPERFICIE (1), GEOPOTENCIAL Y TEMPERATURA A 850 HPA (2) (9/01/2005; 00Z)}
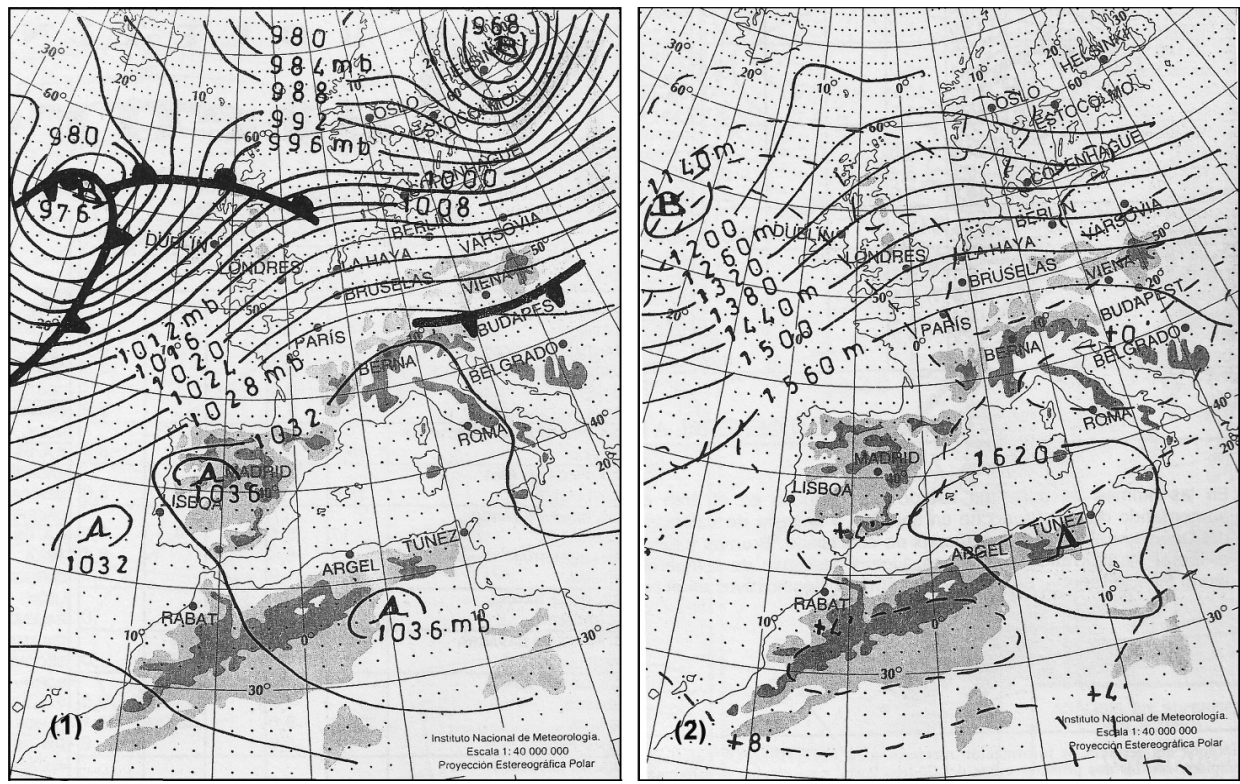

Fuente: Boletín Meteorológico del Instituto Nacional de Meteorología (INM).

Estudios Geográficos, Vol. LXXV, 277, pp. 521-552, julio-diciembre 2014

ISSN: 0014-1496, eISSN: 1988-8546, doi: 10.3989/estgeogr.201416 
máximas generalmente bajas $-13,9^{\circ} \mathrm{C}$ en Llano de Molina y $15,1^{\circ} \mathrm{C}$ en Murcia (UMU)-. En cambio, sí resultó bastante significativa la amplitud térmica diaria en el valle $\left(19,7^{\circ} \mathrm{C}\right.$ en Llano de Molina), donde se alcanzaron los valores nocturnos más extremos. Por otra parte, el sondeo atmosférico de las $00 \mathrm{Z}$ evidenció la existencia de un estrato cálido a $1.000 \mathrm{hPa}(271 \mathrm{~m}$ de altitud), dotado de una temperatura de $7,8{ }^{\circ} \mathrm{C}$ y una humedad relativa de $8 \%$, que contrastaba con los valores térmicos e higrométricos de superficie $\left(2,8{ }^{\circ} \mathrm{C}\right.$ de temperatura y $81 \%$ de HR). Pese a no existir una masa de aire excesivamente fría en niveles medios y bajos de la atmósfera, todo el valle sufrió una potente inversión térmica absoluta, con valores relativamente bajos en superficie $\left(-4,0^{\circ} \mathrm{C}\right.$ en Torres de Cotillas, $-2,6^{\circ} \mathrm{C}$ en Alcantarilla, $0,2{ }^{\circ} \mathrm{C}$ en Murcia y $0,3{ }^{\circ} \mathrm{C}$ en La Alberca). Destaca, no obstante, dentro de esta zona, la especial intensidad que adquirió este fenómeno en el sector norte de la Vega, directamente expuesto a los vientos del N. Así, por ejemplo, el Llano de Molina llegó a registrar durante la noche de este día una fuerte helada $\left(-5,8^{\circ} \mathrm{C}\right)$, cuyos efectos se dejaron notar gravemente en la agricultura. La inversión térmica fue exclusivamente nocturna, como todas las que se producen bajo dominio anticiclónico y presencia de cielos despejados. Con la salida de sol, rápidamente desapareció la capa de inversión térmica, que permaneció de 2,6 a 3,0 horas, tal como por lo general suele ocurrir en valles no muy profundos (Whiteman et al., 2004). Fuera de la zona de influencia de la IT las temperaturas mínimas alcanzadas durante la noche fueron como promedio $8{ }^{\circ} \mathrm{C}$ más altas que en el fondo del valle.

En las jornadas posteriores al día de MIT, 10 y 11 de enero, las altas presiones fueron abandonando la Península de forma progresiva en dirección al centro de la cuenca mediterránea. Su lento desplazamiento hizo que el solar peninsular todavía se viera afectado por el extremo más occidental de la dorsal anticiclónica anotando presiones de hasta $1.032 \mathrm{hPa}$. Ya en niveles altos de la atmósfera, con el transcurso de las horas, se produjo la irrupción de una masa polar marítima, algo más fresca que en jornadas precedentes. Dicha masa de aire terminó recorriendo el Sureste Peninsular el día 11 con una isoterma de 5 ${ }^{\circ} \mathrm{C}$ sobre su vertical a $850 \mathrm{hPa}(00 \mathrm{Z})$, aunque todavía permanecía la situación de bloqueo a las perturbaciones atlánticas.

\section{CONCLUSIONES}

El estudio de las situaciones de inversión térmica más importantes producidas en la Vega Media del Segura durante el período 2000-2011 ha permitido extraer interesantes consideraciones, entre ellas las relativas a las condiciones 
sinópticas que suelen contribuir a su formación y desarrollo: i) predominio anticiclónico en superficie, alimentado en niveles medios y altos con una potente dorsal subtropical marítima, cuya cresta (SO-NE) afecta al sur de la Península Ibérica, con clara advección del O y SO, después de días cálidos y con anomalías térmicas positivas; ii) existencia de una masa de aire subtropical marítima con poca carga higrométrica -desecamiento tras continentalización-, escasa absorción de radiación, y temperatura relativamente cálida en relación a la época del año, con medias de $7,1^{\circ} \mathrm{C}$ a $850 \mathrm{hPa}$.

Estos fenómenos de inversión térmica suelen ser más frecuentes durante la estación invernal, coincidiendo con la larga duración de sus noches (14 horas de media sin radiación solar), un débil flujo de viento en superficie (entre 1,5 y $8,6 \mathrm{~km} / \mathrm{h}$ ), y advecciones de componente O y SO. La temperatura mínima superficial promediada para el conjunto de días de máxima inversión térmica difiere mucho según la posición topográfica de cada observatorio (más de $10{ }^{\circ} \mathrm{C}$ en apenas $116 \mathrm{~m}$ de altitud que separa el fondo del valle respecto a las zonas marginales no afectadas). Se trata, por tanto, de inversiones térmicas con estrato cálido muy bajo, con temperatura media de $12,7^{\circ} \mathrm{C}$ a $490 \mathrm{~m}$ de altitud y una elevada ratio media de descenso térmico desde el estrato cálido a la superficie $\left(-2,01^{\circ} \mathrm{C} / 100 \mathrm{~m}\right)$. Analizada cada una de las SIT se comprueba que los valores de temperatura mínima del día de MIT se sitúan por debajo de lo habitual en las zonas de valle, con aparición de heladas ocasionales (Llano de Molina), y por encima de la media invernal de mínimas diarias en sectores circundantes más altos (Cabezo de la Plata). Las temperaturas máximas absolutas son anormalmente altas para la época del año, lo que determina amplitudes térmicas diarias también muy acusadas y contrastadas dentro del área, con diferencias máximas de casi $10^{\circ} \mathrm{C}$ entre los sectores de mayor y menor exposición a la IT. Finalmente, cabe concluir que el fenómeno de inversión térmica en la Vega Media del Segura no se debe sólo a la pérdida de radiación nocturna, y por ende al enfriamiento radiativo local, sino también a su configuración orográfica en relación con la dirección de los vientos predominantes ( $\mathrm{N}$ y SO) y al drenaje de aire frío catabático en invierno, generalmente procedente de la cercana Sierra de Carrascoy. El presente estudio, no obstante, queda abierto a nuevas y futuras aportaciones que aborden más en detalle la influencia de los vientos de ladera en combinación con los vientos predominantes y los flujos advectivos.

Fecha de recepción: 26 de febrero de 2013.

Fecha de aceptación: 14 de marzo de 2014. 


\section{BIBLIOGRAFÍA}

Albero Sanchís, V. y Català, J. (1965): "Las heladas en la zona naranjera desde el punto de vista meteorológico", en: I Simposio de la Revista de Agroquímica y Tecnología de los Alimentos, Valencia, pp. 23-29.

Bailey, A.; Chase, T. N.; Cassano, J. J. y Noone, D. (2011): "Changing Temperature Inversion Characteristics in the U.S. Southwest and Relationships to Large-Scale Atmospheric Circulation". Journal of Applied Meteorology and Climatology, 50, pp. 1307-1323.

Barr, S. y Orgill, M. M. (1989): "Influence of external meteorology on nocturnal valley drainage winds". Journal of Applied Meteorology, 28, pp. 497-517.

Batlló, J. (2009): "L' anomalia tèrmica de la Plana de Vic, un projecte comú". AUSA, XXIV/163, pp. 217-235.

Bello Fuentes, V. (2008): "Las inversiones térmicas en el Valle Bajo del Henares". Serie Geográfica, 14, pp. 47-60.

Beniston, M. (2006): "Mountain weather and climate: a general overview and a focus on climate change in the Alps". Hydrobiologia, 562, pp. 3-16.

Calvo García-Tornel, F. (1982): Continuidad y cambio en la Huerta de Murcia. Murcia, Real Academia Alfonso X El Sabio.

Capel Molina, J. J. y Romero Macho, Ma . J. (2001): "La dinámica atmosférica en el flanco suroccidental de Europa: La Península Ibérica”. Nimbus, 5-6, pp. 5-20.

Clements, C. B.; Whiteman, C. D. y Horel J. D. (2003): "Cold-air-pool structure and evolution in a mountain basin: Peter Sinks, Utah". Journal of Applied Meteorology, 42, pp. 752-768.

Conesa García, C. y Alonso Sarria, F. (2006): "El Clima de la Región de Murcia", en Conesa García, C. (ed.): El Medio Físico de la Región de Murcia. Murcia, Servicio de Publicaciones, Universidad de Murcia, pp. 95-127.

Daly, Ch.; Conklin, D. R. y Unsworth, M. H. (2009): "Local atmospheric decoupling in complex topography alters climate change impacts". International Journal of Climatology, 30 (12), pp. 1857-1864.

Domínguez Bascon, P. (1995): "Inversiones de temperatura en el valle de Guadalquivir. Un factor climático de gran influencia en el medio ambiente urbano de Córdoba". Anales de Geografía de la Universidad Complutense de Madrid, 15, pp. 281-288.

Dorta Antequera, P. J. (1994): "Las inversiones térmicas en Canarias". Investigaciones Geográficas, 15, pp. 109-123.

García De Pedraza, L. (1962): Las heladas. Madrid, Ministerio de Agricultura. Hojas Divulgadoras, 18

García Martín, F. M. (2012): "Dinámicas de crecimiento de la Huerta de Murcia y similitudes con el Véneto italiano", en: V Jornadas de Introducción a la Investigación de la UPCT. Cartagena, Universidad Politécnica de Cartagena, pp. 21-23.

Geiger, F. (1973): "El Sureste español y los problemas de la aridez". Revista de Geografía, 7/ 1-2, pp. 166-209. 
Gesteiro Araujo, M. y Galán Gallego, E. (1983): "Inversiones térmicas de invierno en la Serranía de Cuenca (Sector Central)", en: VII Coloquio de Geografía. Pamplona, Asociación de Geógrafos Españoles, vol. 1, pp. 109-116.

Gustavsson, T.; Karlsson, M.; Bogren J. y Lindqvist, S. (1998): "Development of temperature patterns during clear nights". Journal of Applied Meteorology, 37, pp. 559-571.

Hernández Navarro, M. L. (1994): “Tipología, génesis y desarrollo de las heladas en el Valle Medio del Ebro. Geographicalia, 31, pp. 95-114

I.N.E. (2012): Censo de población y viviendas, 2011, Madrid, Instituto Nacional de Estadística.

Incecik, S. (1996): "An investigation of the atmospheric conditions in Istanbul leading to air pollution episodes". Atmospheric Environment, 30, pp. 101-111.

Ishikawa, N. (1977): "Studies of radiative cooling at land basins in snowy season". Contributions from the Institute of Low Temperature Sciences, 27 A, pp. 1-46.

Kondo, J. y Okusa, N. (1990): "A simple numerical prediction model of nocturnal cooling in a basin with various topographic parameters". Journal of Applied Meteorology and Climatology, 29, pp. 604-619.

Kukkonen, J.; Pohjola, M.; Sokhi, R. S.; Luhana, L.; Kitwiroon, N.; Rantamaki, M.; Berge, E.; Odegaard, V.; Slørdal, L. H.; Denby, B. y Finardi, S. (2005): "Analysis and evaluation of selected local-scale PM10 air pollution episodes in four European cities: Helsinki, London, Milan and Oslo". Atmospheric Environment, 39, pp. 27592773.

Kuwagata, T. y Sumioka, M. (1991): "The daytime PBL heating process over complex terrain in central Japan under fair and calm weather conditions. Part III: Daytime thermal low and nocturnal thermal high". Journal of the Meteorological Society of Japan, 69, pp. 91-104.

Lillo Carpio, M. (2000): "La Huerta de Murcia como ejemplo de escorrentía derivada inscrita en la llanura de crecida". Papeles de Geografía, 32, pp. 61-75.

Lindkvist, L.; Gustavsson, T. y Bogren, J. (2000): "A frost assessment method for mountainous areas". Agricultural and Forest Meteorology, 102, pp. 51-67.

López Bermúdez, F; Calvo García-Tornel, F. y Morales Gil, A. (1986): Geografía de la Región de Murcia. Barcelona, Ketres Editora, 277 pp.

López Gómez, A. (1975): "Inversión de temperatura entre Madrid y la Sierra de Guadarrama con advección cálida superior". Estudios Geográficos, 36/138-139, pp. 567-604.

Lundquist, J. D. y Cayan, D. R. (2007): "Surface temperature patterns in complex terrain: daily variations and long-term changes in the central Sierra Nevada, California". Journal of Geophysical Research, 112: D11124, doi: 10.1029/2006JD007561, $15 \mathrm{pp}$.

Lundquist, J. D.; Pepin, N. y Rochford, C. (2008): “Automated algorithm for mapping regions of cold-air pooling in complex terrain". Journal of Geophysical Research, 113: D22107. doi: 10.1029/2008JD009879. 
Malek, E.; Davis, T.; Martin, R. S. y Silva, P. J. (2006): "Meteorological and environmental aspects of one of the worst national air pollution episodes (January, 2004) in Logan, Cache Valley, Utah, USA". Atmospheric Research, 79 (2), pp. 108-122.

Martí Ezpeleta, A. (1990): "Las inversiones térmicas en la Depresión de Sariñena". Geographicalia, 27, pp. 105-120.

Martín Vide, J. y Fructuoso Aranda, S. (1993): "Las inversiones térmicas acusadas en el Vallès Oriental”, en: Geografía i Territori. Barcelona, Universitat de Barcelona, pp. 219-226. Colecció Homenatges: Profesor Lluis Casassas.

McKee, T. B. y O'Neal, R. O. (1989): “The role of valley geometry and energy budget in the formation of nocturnal valley winds". Journal of Applied Meteorology, 28, pp. 445-456.

Olcina Cantos, J. y Rico Amorós, A. (1995): "Sequías y golpes de calor en el Sureste Ibérico: Efectos territoriales y económicos". Investigaciones Geográficas, 13, pp. 47-80.

Petkovsek, Z. (1974): "Dissipation of the upper layer of all-day radiation fog in basins". Zbornik Meteorology Hidrol. Radova, 5, pp. 71-74.

Puigdefábregas, J. (1970): “Características de la inversión térmica en el extremo oriental de la depresión interior altoaragonesa". Pirineos, 96, pp. 21-45.

Showalter, A. K. (1953): "A stability index for thunderstorm forecasting". Bulletin of the American Meteorological Society, 34, pp. 250-252.

Trewartha, G. T. (1943): An introduction to weather and climate. New York, McGrawHill Book Company.

Whiteman, C. D. (2000): Mountain Meteorology: Fundamentals and Applications. Oxford, Oxford University Press, 355 pp.

Whiteman, C. D.; Pospichal, B.; Eisenbach, S.; Weihs, P.; Clements, C. B.; Steinacker, R.; Mursch-Radlgruber E y Dorninger, M. (2004): "Inversion Breakup in Small Rocky Mountain and Alpine Basins". Journal of Applied Climatology and Meteorology, 43, pp. 1069-1082.

Xercavins, A. (1989): "Notas sobre el clima y las inversiones térmicas en Les Guilleries", en: Actas del XI Congreso Nacional de Geografía. Madrid, Asociación de Geógrafos Españoles, vol. I-II, pp. 288-295.

Yao, W. y Zhong, S. (2009): "Nocturnal temperature inversions in a small, enclosed basin and their relationship to ambient atmospheric conditions". Meteorology and Atmospheric Physics, 103, pp. 195-210.

\section{RESUMEN}

En el Sureste peninsular son frecuentes las situaciones anticiclónicas alimentadas en invierno por la dorsal subtropical marítima. Bajo estas condiciones, las cuencas y valles fluviales intrabéticos son bastante proclives al desarrollo de inversiones térmicas. Un claro ejemplo lo constituye la Vega Media del Segura en los meses de invierno, especialmente cuando el enfriamiento nocturno del suelo aparece acompañado por mo- 
vimientos de aire anticiclónico subsidente, advecciones cálidas de componente SO a niveles bajos atmosféricos y flujos fríos catabáticos procedentes de la Sierra de Carrascoy. En el presente artículo se analizan tales factores y las situaciones de inversión térmica más importantes registradas en esta Vega durante el período 2000-2011. Para ello se han utilizado los datos de las estaciones meteorológicas y de los sondeos aerológicos disponibles en el área, en particular los relativos a régimen de temperaturas, estratificación térmica vertical e indicadores de estabilidad atmosférica.

Palabras Clave: inversión térmica; anticiclón; advección cálida; Vega Media del Segura; Región de Murcia.

\begin{abstract}
Anticyclonic situations fed by the maritime subtropical dorsal in winter are frequent in the South-east Spain. Under these conditions, the intrabaetic depressions and fluvial valleys are prone to thermal inversions. A clear example is the Middle Valley of the Segura River during winter months, especially when cooling at night appears to be accompanied by anticyclonic subsident air, warm advection SW flows at low atmospheric levels and cold katabatic winds proceeding from Sierra de Carrascoy. Such factors and the most temperature inversion situations registered in the study area during the period 2000-2011 are analyzed in this work. For this purpose data of meteorological stations and aerological soundings (e.g. temperature regime, vertical thermal stratification, indicators of atmospheric stability) were used.
\end{abstract}

KEY WORDS: temperature inversion; anticyclone; warm advection flow; Vega Media del Segura river; Murcia Region.

\title{
RÉSUMÉ
}

Les situations anticycloniques alimentées pendant l'hiver par la dorsale maritime subtropicale sont fréquentes dans le Sud-est espagnol. Dans ces conditions, les bassins et vallées fluviales intrabétiques sont assez enclins au développement d'inversions thermiques. La Vega media del Segura (vallée moyenne du fleuve) constitue un clair exemple pendant les mois d'hiver, spécialement quand le refroidissement nocturne du sol apparaît accompagné par des mouvements d'air anticyclonique subsidente, advections chaudes de direction générale $\mathrm{SO}$ à de bas niveaux atmosphériques et flux froids catabatiques provenant de la Sierra de Carrascoy. Lobjectif de cette recherche est d'analyser ces factors et les situations d'inversion thermique les plus importantes dans l'aire pendant la période 2000-2011. Pour cela, nous avons utilisé les données des stations météorologiques et des sondages aerologiques disponibles dans l'aire, en particulier, les relatifs au régime de températures, de stratification thermique verticale et d'indicateurs de stabilité atmosphérique.

MoTS CLÉS: inversion thermique; anticyclone; advection d'air chaud; Plaine Moyenne du Segure; région de Murcie. 